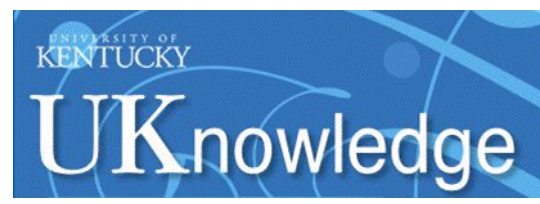

University of Kentucky

UKnowledge

\title{
Very High Density Clumps and Outflowing Winds in QSO Broad- Line Regions
}

\author{
J. A. Baldwin \\ Cerro Tololo Inter-American Observatory, Chile \\ Gary J. Ferland \\ University of Kentucky, gary@uky.edu \\ K. T. Korista \\ University of Kentucky \\ R. F. Carswell \\ Institute of Astronomy, UK \\ F. Hamann \\ University of California - San Diego
}

See next page for additional authors

Follow this and additional works at: https://uknowledge.uky.edu/physastron_facpub

Part of the Astrophysics and Astronomy Commons, and the Physics Commons

Right click to open a feedback form in a new tab to let us know how this document benefits you.

\section{Repository Citation}

Baldwin, J. A.; Ferland, Gary J.; Korista, K. T.; Carswell, R. F.; Hamann, F.; Phillips, M. M.; Verner, D. A.;

Wilkes, Belinda J.; and Williams, R. E., "Very High Density Clumps and Outflowing Winds in QSO Broad-Line Regions" (1996). Physics and Astronomy Faculty Publications. 148.

https://uknowledge.uky.edu/physastron_facpub/148

This Article is brought to you for free and open access by the Physics and Astronomy at UKnowledge. It has been accepted for inclusion in Physics and Astronomy Faculty Publications by an authorized administrator of UKnowledge. For more information, please contact UKnowledge@lsv.uky.edu. 


\section{Very High Density Clumps and Outflowing Winds in QSO Broad-Line Regions}

\section{Digital Object Identifier (DOI)}

http://dx.doi.org/10.1086/177093

\section{Notes/Citation Information}

Published in The Astrophysical Journal, v. 461, p. 664-682.

(c) 1996. The American Astronomical Society. All rights reserved.

The copyright holder has granted permission for posting the article here.

\section{Authors}

J. A. Baldwin, Gary J. Ferland, K. T. Korista, R. F. Carswell, F. Hamann, M. M. Phillips, D. A. Verner, Belinda J. Wilkes, and R. E. Williams 


\title{
VERY HIGH DENSITY CLUMPS AND OUTFLOWING WINDS IN QSO BROAD-LINE REGIONS
}

\author{
J. A. Baldwin, ${ }^{1}$ G. J. Ferland, ${ }^{1,2}$ K. T. Korista, ${ }^{2}$ R. F. Carswell ${ }^{3}$ F. Hamann, ${ }^{4}$ \\ M. M. Phillips, ${ }^{1}$ D. Verner, ${ }^{2}$ Belinda J. Wilkes, ${ }^{5}$ And R. E. Williams ${ }^{1,6}$ \\ Received 1995 July 19; accepted 1995 October 27
}

\begin{abstract}
Intercomparison of the spectra of seven high-luminosity quasi-stellar objects (QSOs) shows that there is a gradation of properties in their broad emission-line regions (BELRs) that can be understood as differing mixtures of different gas components. Six of these seven objects have unusually narrow BELR profiles, which greatly facilitates the disentangling of blends and measuring of weak lines. In the QSO 0207-398, the BELR is made up of at least three kinematically distinct components; its spectrum is in fact a composite of the spectra of the more homogeneous BELRs in the QSOs at either end of our sequence of properties.

This paper focuses on the properties of the line-emitting region in Q0207-398 dubbed component "A," which has sharp $\left(\mathrm{FWHM}=1000 \mathrm{~km} \mathrm{~s}^{-1}\right)$, symmetric line profiles centered at zero velocity. We find that these lines are emitted in very dense $\left(n_{\mathrm{H}} \sim 10^{12.5} \mathrm{~cm}^{-3}\right)$ gas at a characteristic radius $r \sim 10^{17.7}$ $\mathrm{cm}$ from the continuum source and which emits a low-ionization spectrum including strong $\mathrm{Al}$ III $\lambda 1857$.

The second component, "B," in Q0207-398 is the subject of a companion paper. It is characterized by high-ionization lines such as $N$ v $\lambda 1240, O$ VI $\lambda 1034$, and $C$ IV $\lambda 1549$ with profiles that peak at zero velocity but have a blue tail extending out to $-11,000 \mathrm{~km} \mathrm{~s}^{-1}$. It receives about the same incident flux as component $\mathrm{A}$ and therefore may lie at the same distance from the continuum source, but it is significantly less dense $\left(n_{\mathrm{H}} \sim 10^{11} \mathrm{~cm}^{-3}\right)$.

The remaining line emission from $\mathrm{Q} 0207-398$ is attributed to a component " $\mathrm{C}$ " which has reasonably broad $\left(\mathrm{FWHM}=2000 \mathrm{~km} \mathrm{~s}^{-1}\right.$ ), symmetric line profiles centered at zero velocity. Most of the Ly $\alpha$ and C III] $\lambda 1909$ emission comes from this region, but it also contributes to $\mathrm{C}$ IV, $\mathbf{N ~ v}$, and many other lines. The spectrum of component $\mathrm{C}$ is in fact quite similar to that of "normal" QSOs.

We interpret component $\mathrm{A}$ as the dense source for radiatively accelerated, outward flowing gas which we see as component B. Component A may consist of the ablated atmospheres of stars which have strayed too close to the QSO nucleus. In addition, component A's velocity width is essentially the same as that of the stellar population in the nucleus of M87. From the radius and velocity, we infer the presence of a central mass of only $10^{7}-10^{8} M_{\odot}$, if we are measuring virial motions and if the ionizing continuum is isotropic. An alternate possibility is that the narrow single-peaked component A profile comes from a rotating torus/accretion disk, probably seen face-on, in which case it is impossible to measure the central mass.

We discuss the similarity between Mg II-type broad absorption line (BAL) QSOs and Q0207-398, which does not have BALs. In fact, the two Mg II-type BAL objects in our sample have spectra almost exactly like that of Q0207-398, except that component B is seen in absorption rather than in emission.

Subject headings: line: profiles - quasars: emission lines - quasars: individual (Q0207-398)
\end{abstract}

\section{INTRODUCTION}

It is now clear that in many QSOs the broad emissionline regions (BELRs) are far from homogeneous. This shows up as differences between the emission-line profiles of different species (Gaskell 1982; Wilkes 1984; Espey et al. 1989; Corbin 1990), which clearly indicates a connection between velocity and the physical conditions in the emitting gas. These profile differences are easily detectable at fairly low spectral resolution in perhaps a fourth of all $z \sim 2$ QSOs

\footnotetext{
${ }^{1}$ Cerro Tololo Inter-American Observatory, Casilla 603, La Serena, Chile.

${ }^{2}$ Department of Physics and Astronomy, University of Kentucky, Lexington, KY 40506-0055.

${ }^{3}$ Insti tute of Astronomy, Madingley Road, Cambridge CB3 OHA, UK.

${ }^{4}$ Center for Astrophysics and Space Sciences, University of California, San Diego, La Jolla, CA 92093-0111.

${ }^{5}$ Harvard-Smithsonian Center for Astrophysics, 60 Garden Street, Cambridge, M A 02138

${ }^{6}$ Space Telescope Scie nce Institute, 3700 San Martin Drive, Baltimore, MD 21218
}

(Espey et al. 1989). Francis et al. (1992) showed through the technique of principal component analysis that QSO emission-line profiles can in general be thought of as the superposition, in varying proportions, of a few fairly standard components with distinct spectroscopic properties. Similar results were found by Wills et al. (1993) and Brotherton et al. (1994), using a different approach to decomposing the line profiles.

The emission-line spectrum that we see from any part of the BELR is determined by three main parameters: the chemical abundances, the hydrogen density $n_{\mathrm{H}}$, and the ionizing photon flux $\Phi \propto L_{\text {ion }} / r^{2}$ incident on the gas clouds. Because the density and incident flux together set the ionization level in the gas, these two parameters frequently are rolled up together into the single parameter $U \propto L_{\text {ion }} /\left(r^{2} n_{\mathrm{H}}\right)$. But since the incident flux is (for a known luminosity and assuming an isotropically emitting continuum source) a direct measure of the distance of the ionized gas from the continuum source, independent measurements of $n_{\mathrm{H}}$ and $\Phi(\mathrm{H})$ would make it possible to map out the dependence of 
TABLE 1

OBJeCt LIST

\begin{tabular}{|c|c|c|c|c|c|c|}
\hline Object & $z$ & $m_{\lambda}(1550)$ & $L_{\lambda}(1550)^{\mathrm{a}}$ & $L\left(\mathrm{C}_{\mathrm{IV}}\right)^{\mathrm{a}}$ & $\begin{array}{c}W_{\lambda}(\mathrm{C} \text { IV }) \\
(\AA, \text { rest frame })\end{array}$ & $\begin{array}{c}\text { FWHM(C IV) } \\
\left(\mathrm{km} \mathrm{s}^{-1}\right)\end{array}$ \\
\hline Q0000-398 ....... & 2.8331 & 18.56 & $6.8 E+42$ & $4.6 E+44$ & 68.4 & 3500 \\
\hline Q0207-398 ........ & 2.8149 & 17.37 & $2.0 E+43$ & $5.2 E+44$ & 25.9 & 3900 \\
\hline H0335-336 ....... & 2.2566 & 18.71 & $4.2 E+42$ & $2.4 \mathrm{E}+43$ & 5.7 & $1000^{b}$ \\
\hline Q03408-4505 ..... & 2.0178 & 18.59 & $3.9 E+42$ & $2.5 E+43$ & 6.5 & $1200^{b}$ \\
\hline Q1451 + $1017 \ldots \ldots$. & 2.1705 & 19.99 & $1.2 \mathrm{E}+42$ & $9.5 E+43$ & 78.4 & 850 \\
\hline Q1623+268 ....... & 2.5102 & 17.18 & $2.0 \mathrm{E}+43$ & $2.5 \mathrm{E}+44$ & 12.4 & 4900 \\
\hline Q2212-299 ....... & 2.7015 & 17.38 & $1.9 E+43$ & $4.5 E+44$ & 23.8 & 3000 \\
\hline
\end{tabular}

$n_{\mathrm{H}}$ on $r$ in the BELR, to the extent that the source is isotropic. That is the approach we take here.

This paper is in part a comparative study of high-quality optical spectra of seven QSOs, some of which show these profile differences and some of which do not. Six of these QSOs have unusually narrow emission lines, which greatly facilitates the untangling of blends and of different velocity components. However, our detailed analysis will concentrate on one especially interesting QSO, 0207-398, which has three very distinct kinematic components contributing to its emission lines. We will suggest a possible connection to the two broad absorption line (BAL) QSOs in our sample and contrast it to several objects which seem to have more homogeneous BELRs.

A companion paper reports other aspects of this same study. Ferland et al. (1996, hereafter Paper II) show that the $\mathrm{N} \mathrm{v} \lambda 1240 / \mathrm{He}$ II $\lambda 1640$ intensity ratio is a good indicator of the metallicity in BELRs.

\section{OBSERVATIONAL MATERIAL}

\subsection{Selection of QSOS}

Four of the QSOs studied here (Q0000-398, Q2212-299, Q1623 + 268, and Q0207-398) were chosen because there already existed high-resolution spectra of them covering the rest wavelength range from about $912 \AA$ to the Ly $\alpha$ emission line. This region is riddled by $L y \alpha$ forest absorption lines, so that reasonably high signal-to-noise ratio, high-resolution spectra are necessary to have any chance of detecting weak emission lines. We had noticed some time ago that Q0207-398 has detectable C III $\lambda 977$ emission in this region, and we wanted to compare it to other QSOs of similar redshift and luminosity.

Table 1 lists redshifts, magnitudes, and luminosities for each of these QSOs, while Table 2 gives the references for the previously published spectra we have used as well as relevant information about the new observations.

TABLE 2

SPECTRA

\begin{tabular}{|c|c|c|c|c|c|c|}
\hline Object & Date (UT) & Telescope & Detector & Rest $\lambda$ Range & Rest FWHM & Reference \\
\hline \multirow[t]{4}{*}{ Q0000-398 ....... } & $1990 \mathrm{Jul} 24$ & CTIO & GEC & $860-1712$ & 3.0 & 1 \\
\hline & $1990 \mathrm{Jul} 25$ & CTIO & GEC & $1638-2499$ & 3.0 & 1 \\
\hline & 1990 Oct 27 & CTIO & Reticon & 1335-2019 & 1.4 & 1 \\
\hline & 1979 Nov 25 & AAT & IPCS & $886-1689$ & $0.6-1.0$ & 2 \\
\hline \multirow[t]{5}{*}{ Q0207-398 ....... } & 1990 Jul 24 & CTIO & GEC & $863-1720$ & 3.0 & 1 \\
\hline & $1990 \mathrm{Jul} 25$ & CTIO & GEC & $1646-2511$ & 3.0 & 1 \\
\hline & 1990 Jul 27 & CTIO & Reticon & $1342-2028$ & 1.4 & 1 \\
\hline & 1979 Nov 26 & AAT & IPCS & $911-1626$ & $0.6-1.0$ & 2 \\
\hline & 1977 Nov 17 & AAT & IPCS & $843-1520$ & 0.4 & 3 \\
\hline \multirow[t]{5}{*}{$\mathrm{H} 0335-336 \ldots \ldots$} & 1983 Oct 30 & CTIO & GEC & $2166-2922$ & 2.6 & 4 \\
\hline & 1983 Oct 31 & CTIO & GEC & $1642-2026$ & 1.3 & 4 \\
\hline & 1983 Nov 1 & CTIO & GEC & $1768-2148$ & 1.3 & 4 \\
\hline & 1983 Nov 1 & CTIO & 2D-Frutti & $1104-1679$ & 0.6 & 5 \\
\hline & 1983 Nov 1 & CTIO & GEC & $1000-1930$ & 1.4 & 1 \\
\hline 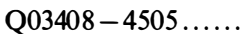 & 1983 Oct 30 & CTIO & GEC & $1110-3220$ & 4.1 & 6 \\
\hline \multirow{3}{*}{$\mathrm{Q} 1451+1017 \ldots \ldots$} & 1986 Mar 2 & CTIO & GEC & $2417-2972$ & 1.9 & 7 \\
\hline & 1986 Mar 10 & CTIO & 2D-Frutti & $1105-2212$ & 2.2 & 7 \\
\hline & 1992 Feb 11 & CTIO & Reticon & $1013-2356$ & 2.8 & 1 \\
\hline \multirow[t]{3}{*}{$\mathrm{Q} 1623+268 \ldots \ldots \ldots$} & 1990 Jul 24 & CTIO & GEC & $939-1870$ & 3.2 & 1 \\
\hline & $1990 \mathrm{Jul} 25$ & CTIO & GEC & $1789-2729$ & 3.2 & 1 \\
\hline & 1991 Apr 21 & CTIO & Reticon & $922-2142$ & 2.5 & 1 \\
\hline \multirow[t]{4}{*}{ Q2212-299 ....... } & 1990 Jul 24 & CTIO & GEC & $890-1773$ & 3.0 & 1 \\
\hline & 1990 Jul 25 & CTIO & GEC & $1696-2588$ & 3.0 & 1 \\
\hline & 1990 Oct 27 & CTIO & Reticon & $1384-2090$ & 1.4 & 1 \\
\hline & 1981 Sep 29 & AAT & IPCS & $923-1931$ & $0.6-1.0$ & 2 \\
\hline
\end{tabular}

REFERENCES--11) New observation; (2) Wilkes 1984; (3) R. F. Carswell, A. Boksenberg, \& M. G. Smith unpublished; (4) Hartig \& Baldwin 1986; (5) D. A. Turnshek, R. J. Weymann, G. F. Hartig, \& J. A. Baldwin unpublished; (6) J. Baldwin, B. Peterson, B. Boyle, \& T. Shanks unpublished; (7) Baldwin et al. 1988. 
To calibrate the existing high-resolution spectra in the blue, and to extend the coverage into the red, we obtained additional spectra with 5-9 $\AA$ resolution in the observed frame. These have good spectrophotometric accuracy, so that we can adequately measure continuum shapes and relative intensities of widely separated emission lines. The final wavelength coverage in the rest frame of each QSO is approximately $\lambda \lambda 900-2500$.

As soon as we reduced our first set of low-dispersion spectra, it became apparent that Q0207-398 is unusual not only because C III $\lambda 977$ can be detected, but also because of the presence of strong lines of $\mathrm{Al}$ III and other species in the vicinity of C III] $\lambda 1909$. In fact, at the time Q0207-398 was reduced, there were only two other QSOs known to us which had spectra closely resembling that of Q0207-398 in the $\lambda 1909$ region: the broad absorption line (BAL) objects H0335 - 334 (Hazard et al. 1984) and Q03408 - 4505 (Boyle et al. 1990). Others are now known (see Weymann et al. 1991), and many of these are also BAL QSOs.

In order to make a better comparison, we observed $\mathrm{H} 0335-334$ in the rest wavelength range $\lambda \lambda 1050-1680$ to complement existing data at longer wavelengths (we could not observe C III $\lambda 977$ in this object because it falls below the atmospheric cutoff). In the case of Q03408-4505, we used an existing spectrum from unpublished work by $\mathrm{J}$. Baldwin, B. Peterson, B. Boyle, and T. Shanks. This covers the observed wavelength range $\lambda 23250-9700$ (rest wavelength range $\lambda \lambda 1110-3220)$ at $12 \AA$ resolution with good spectrophotometric accuracy.

Finally, since Q0207-398, H0335-334, and Q03408-4505 all have exceptionally narrow emission lines, we also obtained an additional blue spectrum of the narrow-line QSO $1451+1017$. This supplements data taken by Baldwin et al. (1988).

\subsection{Observing Procedure}

The new spectra were obtained with the Ritchey-Chrétien (RC) spectrograph on the Cerro Tololo Inter-American Observatory (CTIO) $4 \mathrm{~m}$ telescope. We initially used a GEC CCD with 576 pixels along the dispersion to observe with two grating setups which together covered the wavelength range $\lambda \lambda 3296-9580$ with $12 \AA$ resolution. The later observations were made with a Reticon CCD which has 1200 pixels in the dispersion direction and much higher blue response than the GEC, using grating settings covering either $\lambda 25123-7739$ at $5 \AA$ resolution or (for $Q 1623+268$ ) $\lambda \lambda 3240-7520$ at $9 \AA$ resolution.

These new data were combined with previously existing spectra taken either at the CTIO $4 \mathrm{~m}$ or the Anglo Australian Telescope (AAT) (see Table 2).

For each object, most of the observations were taken through a narrow $\left(\sim 1^{\prime \prime} 5\right)$ slit, but both blue and red lowresolution spectra were obtained at CTIO on photometric nights through a $7^{\prime \prime}$ or $10^{\prime \prime}$ slit in order to obtain accurate (to about $5 \%$ ) spectrophotometry over the full optical passband. Each narrow-slit spectrum (including those from the AAT) was then binned heavily in wavelength and divided into the appropriate wide-slit spectrum to derive a normalizing function at each wavelength. The unbinned narrowslit spectra were then multiplied by these normalizing functions to put them onto similar flux scales, after which they were coadded, weighting them so that at any given wavelength only the highest resolution data contributed to the final summed spectrum. Table 1 lists the monochro- matic magnitudes at the position of the redshifted $\mathrm{C}$ IV line, measured from the final coadded spectra.

\subsection{Emission-Line Measurements}

The overall spectrum of each object is shown in Figure 1. We used the highest resolution data available in each wavelength range, so that while the resolution is only around 500 $\mathrm{km} \mathrm{s}^{-1}$ to the red of $\mathrm{Ly} \alpha$, it is much higher to the blue with the result much of the apparent noise at the blue end is actually just the $L y \alpha$ forest absorption lines.

The region between Ly $\alpha$ and C III] $\lambda 1909$, after subtracting the continuum, is shown in Figure 2. In order to measure the strengths of the numerous, frequently blended emission lines in this region, we fitted synthetic spectra constructed separately for each QSO. We used the profiles of different strong emission lines as templates which were then shif ted to the correct wavelengths and added while varying the relative intensities of different multiplets until a best fit was achieved. This was done as an interactive procedure, with goodness of fit judged by visually examining a residual plot.

The wavelengths and relative intensities within multiplets as used in these fits are listed in Table 3 and are also indicated by the position and size of the tick marks under each spectrum in Figure 2. For many of the multiplets, we assumed that the relative strength of each line is proportional to the Einstein $A$-value multiplied by the upper level's statistical weight, which will be true if the sublevels are indeed populated according to their statistical weights

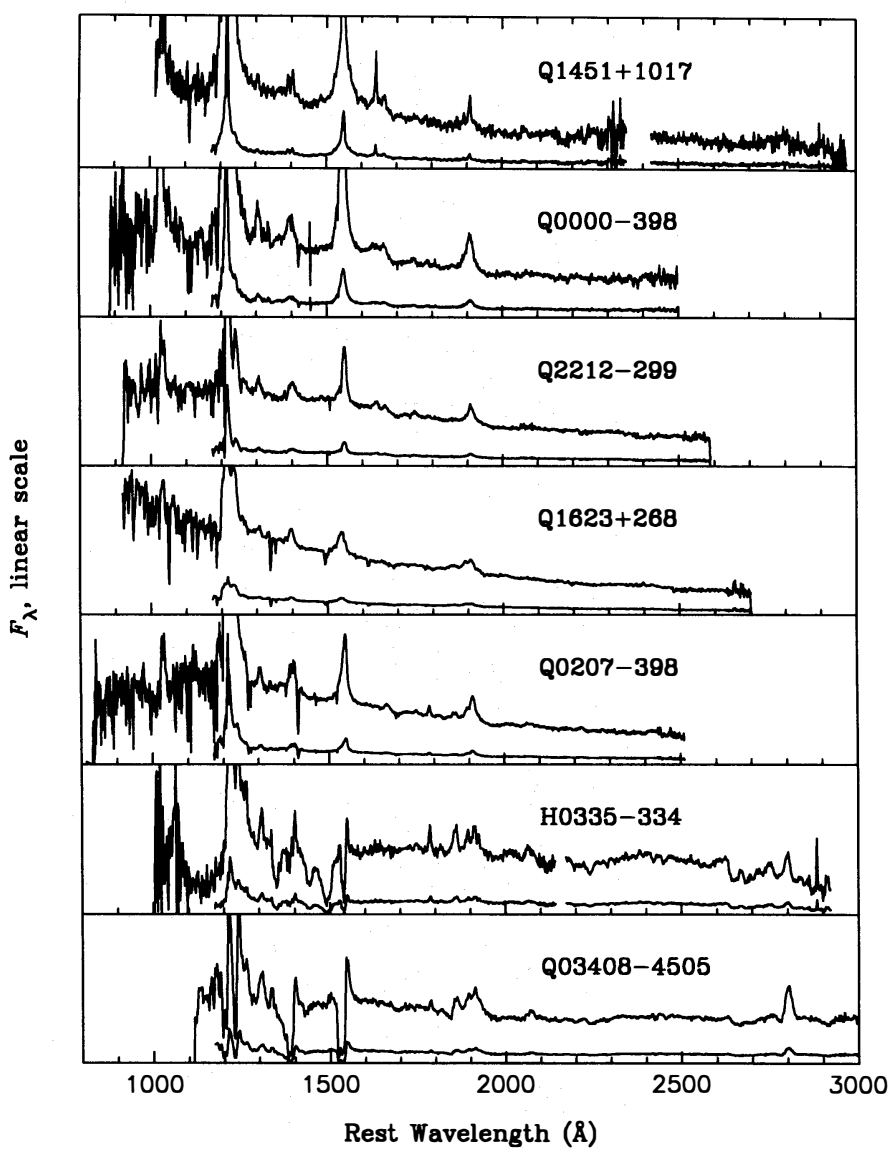

FIG. 1.-Overall rest-frame spectrum of each object in our sample. For each QSO, the same data are plotted on two vertical scales in order to show both the line peaks and the detail at the continuum level. 


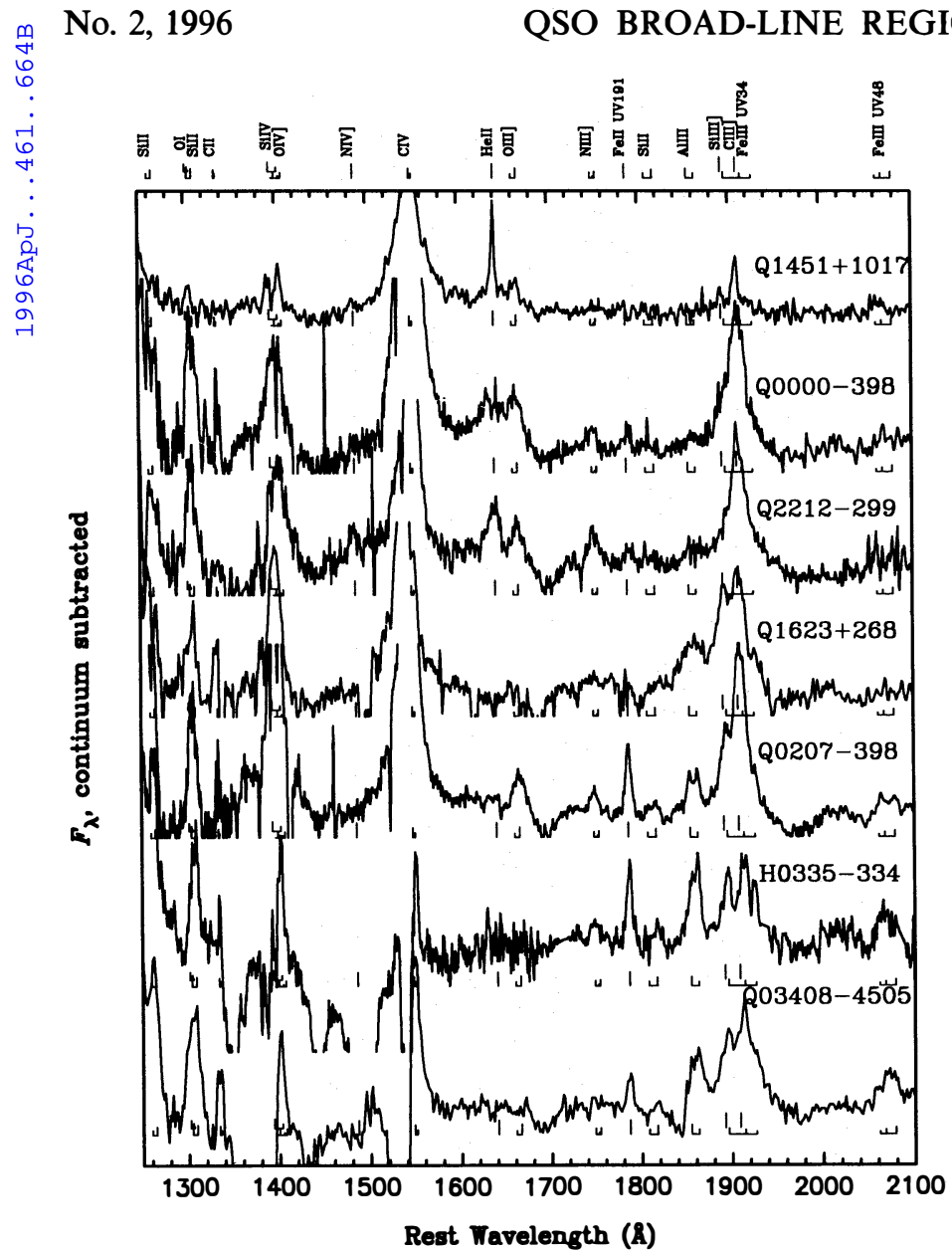

FIG. 2.-The region between Ly $\alpha$ and C III] $\lambda 1909$, after subtracting the continuum. The tick marks show the positions of the different lines (Table 3) fitted to the observed profiles. The heights of the ticks show the relative strengths, within multiplets, used in the fitting.

and if the gas is optically thin in the line in question. If the gas is optically thick in a particular line, the lines in the multiplet will all have the same intensity; this was assumed to be the case for O vi $\lambda 1034, \mathrm{~N}$ v $\lambda 1240, \mathrm{O}_{\mathrm{I}} \lambda 1303, \mathrm{Si}$ IV $\lambda 1397, \mathrm{C}$ IV $\lambda 1549, \mathrm{Fe}$ II UV 191, and Al III $\lambda 1857$. For the Fe III UV 34 multiplet, we used the individual intensity ratios measured from the spectrum of $\mathrm{H} 0335-336$, where the components are clearly resolved.

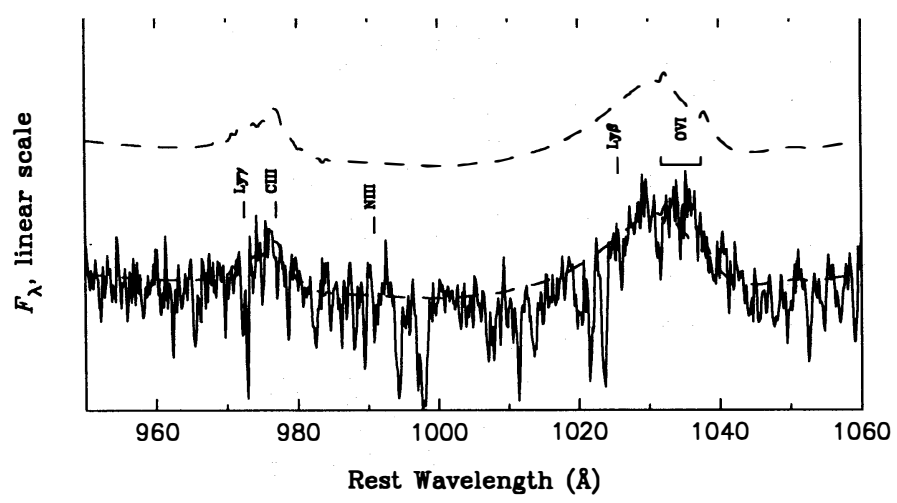

Fig. 3.-Blow up of the spectral region including C III $\lambda 977$, Ly $\gamma$ and the Ly $\beta, O$ y $\lambda 1034$ blend in Q0207-398. A dashed line showing the fitted profiles is shown superimposed on the observed spectrum and then again shifted upward by $\frac{1}{3}$ of the figure height. The tick marks show the expected location of several emission lines for which strengths or upper limits are derived.
TABLE 3

LiNes USED IN ProfiLe Fits

\begin{tabular}{|c|c|c|}
\hline Line & $\lambda$ & Statistical Weight \\
\hline $\operatorname{Ly} \gamma \ldots \ldots \ldots$ & 972.54 & 1.0 \\
\hline C III] 977 . & 977.03 & 1.0 \\
\hline $\mathrm{N}$ III $991 \ldots \ldots \ldots \ldots \ldots$ & 990.98 & 1.0 \\
\hline $\operatorname{Ly} \beta \ldots \ldots \ldots \ldots \ldots$ & 1025.72 & 1.0 \\
\hline \multirow{2}{*}{ O vi $1034 \ldots \ldots \ldots \ldots \ldots$} & 1031.95 & 0.50 \\
\hline & 1037.63 & 0.50 \\
\hline Ly $\ldots \ldots \ldots \ldots \ldots \ldots$ & 1215.67 & 1.0 \\
\hline \multirow[t]{2}{*}{ N v $1240 \ldots \ldots \ldots \ldots$} & 1238.81 & 0.50 \\
\hline & 1242.80 & 0.50 \\
\hline \multirow[t]{3}{*}{ Si II $1263 \ldots \ldots \ldots \ldots \ldots$} & 1260.42 & 0.33 \\
\hline & 1264.73 & 0.60 \\
\hline & 1265.02 & 0.07 \\
\hline \multirow[t]{3}{*}{ O I 1303} & 1302.17 & 0.33 \\
\hline & 1304.87 & 0.33 \\
\hline & 1306.04 & 0.33 \\
\hline \multirow{2}{*}{ Si II $1307 \ldots \ldots \ldots \ldots . . . .}$. & 1304.37 & 0.33 \\
\hline & 1309.28 & 0.67 \\
\hline \multirow[t]{3}{*}{ C II $1335 \ldots \ldots \ldots \ldots \ldots$} & 1334.53 & 0.60 \\
\hline & 1335.66 & 0.32 \\
\hline & 1335.71 & 0.07 \\
\hline \multirow{2}{*}{ Si Iv $1397 \ldots \ldots \ldots \ldots \ldots$} & 1393.76 & 0.50 \\
\hline & 1402.77 & 0.50 \\
\hline \multirow{4}{*}{ O Iv] $1402 \ldots \ldots \ldots \ldots$} & 1397.21 & 0.02 \\
\hline & 1399.78 & 0.41 \\
\hline & 1404.79 & 0.16 \\
\hline & 1407.39 & 0.41 \\
\hline $\left.\mathrm{N}_{\text {Iv }}\right] 1486 \ldots \ldots \ldots \ldots$ & 1486.50 & 1.00 \\
\hline \multirow{2}{*}{$\mathrm{C}$ iv $1549 \ldots \ldots \ldots \ldots \ldots$} & 1548.20 & 0.50 \\
\hline & 1550.77 & 0.50 \\
\hline He II $1640 \ldots \ldots \ldots \ldots$ & 1640.72 & 1.00 \\
\hline \multirow{2}{*}{$\mathrm{O}$ III] $1665 \ldots \ldots \ldots \ldots \ldots$} & 1660.80 & 0.29 \\
\hline & 1666.14 & 0.71 \\
\hline \multirow[t]{3}{*}{$\left.\mathrm{N}_{\text {III }}\right] 1750 \ldots \ldots \ldots \ldots$} & 1748.65 & 0.41 \\
\hline & 1752.16 & 0.14 \\
\hline & 1754.00 & 0.45 \\
\hline Fe II UV191 $1787 \ldots \ldots$ & 1786.7 & 1.00 \\
\hline \multirow{3}{*}{ Si II $1814 \ldots \ldots \ldots \ldots \ldots$} & 1808.00 & 0.33 \\
\hline & 1816.92 & 0.60 \\
\hline & 1817.45 & 0.07 \\
\hline \multirow[t]{2}{*}{$\mathrm{Al}$ III $1857 \ldots \ldots \ldots \ldots$} & 1854.72 & 0.50 \\
\hline & 1862.78 & 0.50 \\
\hline Si III] $1892 .$. & 1892.03 & 1.00 \\
\hline C III] $1909 \ldots \ldots \ldots \ldots \ldots$ & 1908.73 & 1.00 \\
\hline \multirow[t]{3}{*}{ Fe III UV34 $1909 \ldots \ldots$} & 1895.46 & 0.39 \\
\hline & 1914.06 & 0.46 \\
\hline & 1926.30 & 0.15 \\
\hline \multirow[t]{3}{*}{ Fe III UV48 $2071 \ldots \ldots$} & 2061.55 & 0.20 \\
\hline & 2068.24 & 0.33 \\
\hline & 2078.99 & 0.47 \\
\hline \multirow[t]{2}{*}{$\mathrm{N}$ II] $2142 \ldots \ldots \ldots \ldots$} & 2139.7 & 0.29 \\
\hline & 2143.5 & 0.71 \\
\hline \multirow[t]{2}{*}{ Mg II $2798 \ldots \ldots \ldots \ldots . .}$. & 2795.53 & 0.67 \\
\hline & 2802.70 & 0.33 \\
\hline
\end{tabular}

Figure 3 shows a blowup of the spectral region of Q0207-398 which includes the C III $\lambda 977$, Ly $\gamma$ blend and the Ly $\beta, O$ vi $\lambda 1034$ blend. The dashed line shows the fits to the two emission features, using the relative intensities and line profiles (see $\$ 4.1$ ) given in Table 4. The regions around Ly $\alpha$ and $\mathrm{He}$ II $\lambda 1640$ can be seen in more detail in Figure 1 of Paper II.

The details of the fitting procedure for the individual objects are given in Appendix A (except for Q0207-398, which is described in $\S 4$ below). The profiles of the strongest emission lines for each object are shown in Figure 4, on a consistent velocity scale for easy intercomparison. The derived line intensities are presented in Table 4. In the cases of $\mathrm{Q} 0207-398$ and $\mathrm{Q} 1623+268$, results are given in 
TABLE 4

FitTed EMission Line Strengths

\begin{tabular}{|c|c|c|c|c|c|c|c|c|c|c|}
\hline \multirow[t]{2}{*}{ Line } & \multirow[t]{2}{*}{ Q1451 } & \multirow[t]{2}{*}{ Q0000 } & \multirow[t]{2}{*}{ Q2212 } & \multicolumn{2}{|c|}{ Q1623 } & \multicolumn{3}{|c|}{ Q0207 } & \multirow[t]{2}{*}{ H0335 } & \multirow[t]{2}{*}{ Q03408 } \\
\hline & & & & A & $\mathrm{B}$ & A & B & $\mathrm{C}$ & & \\
\hline Ly $\gamma 973$ & & & & & & $<0.23$ & $<0.22$ & 0.27 & & \\
\hline $\mathrm{C}_{\text {III }} 977$ & & $<0.20$ & $<0.50$ & $<0.44$ & $<4.48$ & 0.29 & $<0.12$ & $<0.15$ & & \\
\hline N III 991 & & & & & & $<0.23$ & $<0.28$ & $<0.27$ & & \\
\hline $\operatorname{Ly} \beta 1026$ & & $\leq 0.15$ & $\leq 0.3:$ & $<0.42$ & $<4.33$ & $<0.45$ & $<0.27$ & $<0.30$ & & \\
\hline () VI 1034 & & 0.53 & 0.58 & 0.85 & $<8.67$ & $\leq 0.70$ & 1.13 & $<1.10$ & & \\
\hline Ly $\alpha 1216$ & 2.31 & 3.57 & 4.35 & 1.53 & 11.50 & $<4.86$ & $<2.83$ & 10.34 & & \\
\hline N v 1240 & 0.16 & 0.31 & 0.58 & 1.51 & $<4.50$ & $<1.14$ & 1.11 & 1.16 & 1.0: & 3.3: \\
\hline Si II 1263 & $<0.13$ & 0.11 & 0.13 & & & 0.55 & $<0.20$ & $<0.20$ & $0.7:$ & 0.6 : \\
\hline O I 1303 & 0.06 & $<0.05$ & $\leq 0.21$ & $<0.08$ & $2.07 ?$ & $<0.23$ & $<0.01$ & $<0.20$ & 0.2 : & 0.2 : \\
\hline Si II 1307 & $<0.03$ & 0.15 & 0.21 & $<0.24$ & $<2.17 ?$ & 0.48 & $<0.01$ & $<0.20$ & $0.8:$ & 0.6 : \\
\hline C II 1335 & $<0.01$ & $<0.12$ & $<0.02$ & $<0.08$ & $<0.83$ & 0.18 & $<0.04$ & $<0.10$ & 0.2 : & 0.26 \\
\hline Si IV 1397 & 0.13 & 0.1 : & $\leq 0.3$ & $<0.21$ & $4.97 ?$ & $<0.34$ & $<0.24$ & 1.13 & 1.7: & 1.11 \\
\hline O IV] 1402 & $<0.05$ & 0.06 : & $0.3:$ & $<0.64$ & $<1.50 ?$ & $<0.03$ & $<0.02$ & $<0.30$ & $<0.37$ & $<0.17$ \\
\hline N IV] 1486 & 0.02 : & $<0.02$ & 0.07 & $<0.05$ & $<0.50$ & $<0.45$ & $<0.06$ & $<0.03$ & & $<0.13$ \\
\hline C IV 1549 & 1.00 & 1.00 & 1.00 & 1.00 & $<1.00$ & $<1.00$ & 1.00 & 1.00 & 1.00 & 1.0: \\
\hline He II 1640 & 0.25 & 0.06 & 0.16 & $<0.06$ & $<0.01$ & $<0.14$ & $<0.12$ & $<0.07$ & $<0.20$ & $<0.06$ \\
\hline O)III] 1665 & $0.08:$ & 0.09 & 0.10 & $<0.05$ & $<0.33$ & 0.13 & $<0.10$ & $<0.20$ & $<0.21$ & $<0.06$ \\
\hline N III] 1750 & $<0.01$ & 0.04 & 0.07 & 0.09 & $<0.42$ & $<0.11$ & 0.10 & $<0.09$ & 0.22 & $<0.10$ \\
\hline Fe 111787 & $<0.02$ & 0.02 & 0.05 & $<0.05$ & $<0.50$ & 0.23 & $<0.01$ & $<0.09$ & 0.54 & 0.14 \\
\hline Si II 1814 & $<0.01$ & 0.01 & 0.05 & $<0.05$ & $<0.50$ & 0.08 & $<0.02$ & $<0.04$ & 0.21 & $<0.10$ \\
\hline Al III 1857 & $<0.01$ & 0.02 & 0.06 & $<0.05$ & 2.20 & 0.22 & $<0.02$ & $<0.20$ & 1.19 & 0.47 \\
\hline Si III] 1892 & 0.03 & $<0.04$ & $<0.05$ & & 2.45 & $<0.13$ & $<0.02$ & $<0.03$ & $<0.22$ & $<1.17$ \\
\hline C III] 1909 & 0.14 & 0.25 & 0.44 & $<0.82$ & 3.90 & $<0.32$ & $<0.16$ & 0.68 & $<0.49$ & $<1.17$ \\
\hline Fe III 1909 & & $<0.12$ & & & & 0.48 & $<0.15$ & $<0.57$ & 3.58 & 2.3: \\
\hline Fe III? 2071 & $\leq 0.04$ & $<0.03$ & 0.09 & $<0.08$ & $<0.83$ & $<0.18$ & $<0.06$ & 0.15 & 0.72 & 0.48 \\
\hline $\mathrm{N} \mathrm{II]} 2142$ & $<0.03$ & $<0.02$ & 0.03 & $<0.16$ & $<1.33$ & $<0.05$ & $<0.03$ & $<0.06$ & $<0.14$ & $<0.06$ \\
\hline C II] 2336 & $<0.03$ & $<0.01$ & $<0.05$ & $<0.16$ & $<1.33$ & $<0.05$ & $<0.03$ & $<0.06$ & $<0.14$ & $<0.06$ \\
\hline Mg II 2798 & 0.19 & & & & & & & & 1.35 & 1.7: \\
\hline $\mathrm{F}(\mathrm{C} \mathrm{IV})$ & $1.1 \mathrm{E}-14$ & $3.1 \mathrm{E}-14$ & $3.3 \mathrm{E}-14$ & $2.2 \mathrm{E}-14$ & $<2.1 \mathrm{E}-15$ & $<8.4 \mathrm{E}-15$ & $1.7 \mathrm{E}-14$ & $1.8 \mathrm{E}-14$ & $2.7 \mathrm{E}-15$ & $3.6 \mathrm{E}-15$ \\
\hline
\end{tabular}

NoTE.-Values with no additional symbols represent our best measurement of a line that we think really is present. " <" indicates upper limit where no line is seen or (for Q0207 or Q1623) that the contribution to the component is less than the listed value. " $\leq$ " indicates upper limit where a line appears to be present but is too weak or too blended to measure. "?" indicates uncertain identification. Colon after a number indicates uncertainty greater than factor of two. Blank entries are cases where we cannot determine anything meaningful about the line in question.

separate columns for the individual kinematic components described below. Within each column the line strengths have been normalized to the strength (or upper limit) for C IV 21549 . The normalizing factor (in units of ergs $\mathrm{cm}^{-2}$ $\mathrm{s}^{-1}$ in the observed frame) is listed in the bottom row of the table.

Upper limits were determined by increasing the line strength slowly until significant negative fluctuations appeared in the residual plot. This is also a reasonable estimate of the uncertainty in the measured strength of nearby lines. For unblended lines against a smooth continuum, this corresponds very roughly to the line peak exceeding a continuum noise of about $2 \sigma$ after "eyeball" smoothing to a resolution equal to the narrowest features of interest (approximately the resolution shown in Fig. 1). However, the uncertainties are increased significantly by uncertainties in the continuum placement and in the accuracy of the modeling of other lines in a blend, and/or by failure of the template to describe precisely the observed profile. The best rule of thumb for assessing the uncertainties in the line strengths given in Table 4 is to use the listed upper limits for nearby lines in the same spectrum. Failing that, typical uncertainties are $\sim 10 \%$ of the $C$ IV strength for lines to the redward of Ly $\alpha$ emission and 2-3 times worse for lines blueward of Ly $\alpha$ emission.

\section{COMPARISON OF SPECTRA}

\subsection{Overview}

In Figures 1 and 2 we have shown the spectra in what we feel is a logical progression of properties. We consider the upper three objects to have fairly "normal" emission-line spectra except for the fact that the emission lines are rather narrow (1000-3000 $\mathrm{km} \mathrm{s}^{-1}$ FWHM). At the bottom of the figures, we have placed two BAL QSOs with virtually identical emission-line spectra. In the region from 1700 to 2000 $\AA$, these have very obvious differences from the top three spectra; there is a fairly strong line at $1787 \AA$, strong Al III $\lambda 1857$ emission, and considerable substructure in the $\lambda 1909$ emission feature. In the middle, we place Q1623+268 and Q0207-398, which seem to be a composite of the distinctly different spectral types above and below them and also to have as an additional distinctive feature extended blue tails on their C IV $\lambda 1549$ lines (see Fig. 4). There are particularly obvious similarities between the bottom two spectra and that of Q0207-398; the main difference is that the lines in Q0207-398 which have extended blue tails in emission are the ones now seen in absorption in the BAL spectra. $\mathrm{Q} 1623+268$ appears to have a very similar spectrum, except that the details have been washed out by the much greater widths of the line profiles.

We describe this sequence of properties in greater detail below.

\section{2. $\mathrm{Al}$ III $\lambda 1857$ and the $\lambda 1909$ Region}

We were particularly struck by the great similarity in this region between the spectrum of Q0207-398 and those of H0335-398 and Q03408-4505. There can be no doubt that the double-peaked feature near $\lambda 1857$ in all three spectra arises from the $\mathrm{Al}$ III $\lambda 1854.7,1862.8$ doublet; the wavelength separation is exact. The two lines should have a 


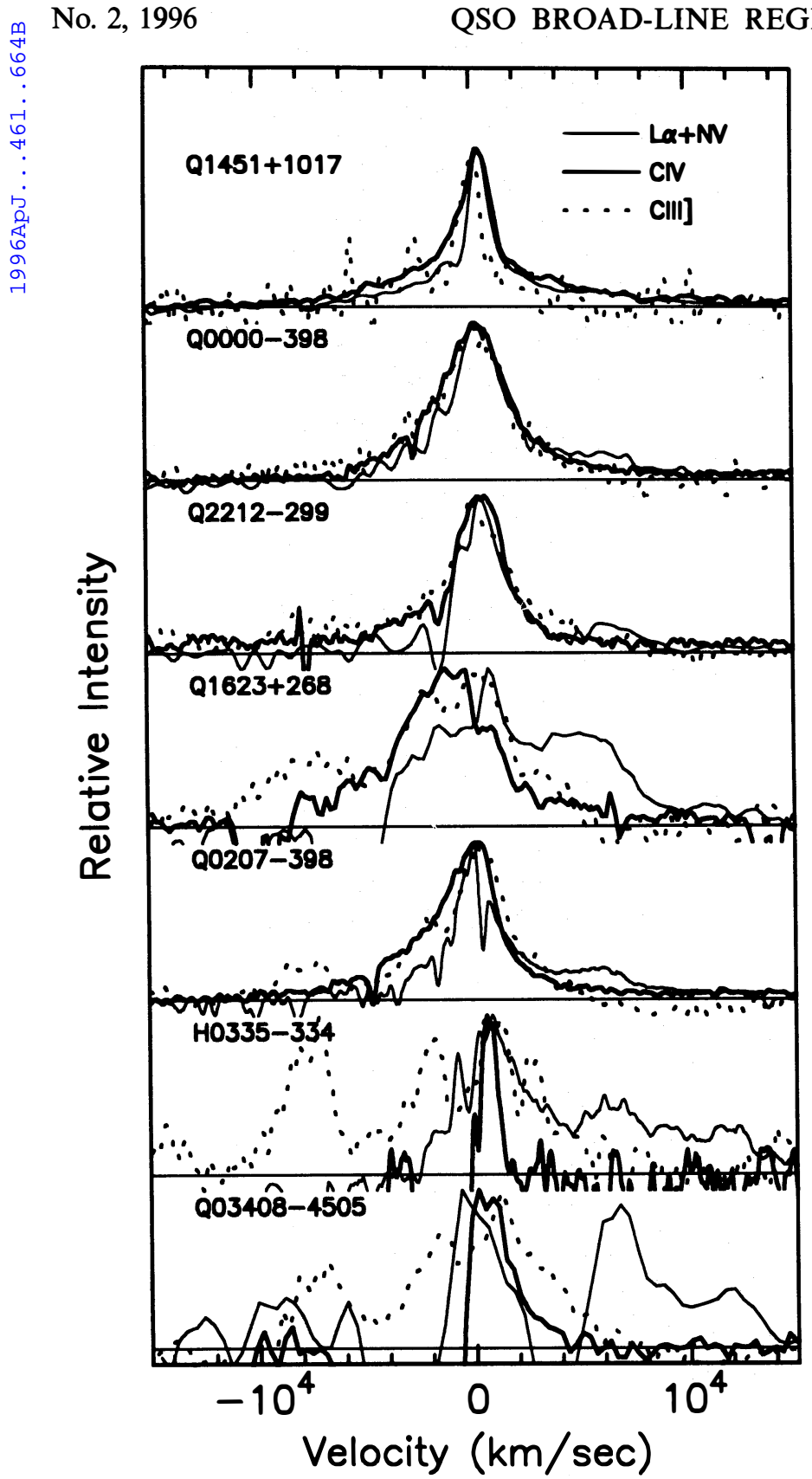

FIG. 4.-The profiles of the strongest emission lines for each object, scaled to the same peak intensity and overplotted on a consistent velocity scale for easy intercomparison.

$2: 1$ intensity ratio if they are optically thin and collisionally excited. In fact, in all three cases they are in a $1: 1$ ratio, showing that the lines are optically thick.

From this same spectrum of H0335-334, Hartig \& Baldwin (1986) concluded that the three peaks between 1895 and $1927 \AA$ are from the Fe III UV 34 multiplet, and that C III] $\lambda 1909$ and $\mathrm{Si}$ III] $\lambda 1892$ are not detected. Weymann et al. (1991) have since found that BAL QSOs with strong $\mathrm{Mg}$ II and $\mathrm{Al}$ III absorption also tend to have $\mathrm{Fe}$ III emission which is stronger than $\mathrm{C}$ III] $\lambda 1909$.

We will show in $\S 4.2 .2$ that $\mathrm{Fe}$ III is also a strong contributor to the $\lambda 1909$ blend in Q0207-398, although in this case half the flux in the $\lambda 1909$ blend comes from C III] $\lambda 1909$.

$\mathrm{Q} 1623+268$ is the other object with strong $\mathrm{Al}$ III. Here there is no clear evidence for Fe III. The $\lambda 1909$ blend can be fitted satisfactorily using just $\mathrm{Si}$ III] and $\mathrm{C}$ III] in a nearly $1: 1$ intensity ratio. However, the lines in this object are so broad and asymmetrical that the Fe III multiplet could easily be as strong as Al III without being detectable.

A weakening first of $\mathrm{C}$ III] and then of $\mathrm{Si}$ III] at progressively higher densities is expected from just considering the critical densities of these lines for collisional de-excitation $\left(\tau n_{\text {crit }} \approx 4.2 \times 10^{9} \mathrm{~cm}^{-3}\right.$ for C III] and $\tau n_{\text {crit }} \approx 1.1 \times 10^{11}$ $\mathrm{cm}^{-3}$ for Si III], $\tau>1$ ). However, Figure 2 shows that the $\mathrm{Al}$ III and $\mathrm{Fe}$ III lines seem to get stronger at the same time, relative to $\mathrm{C}$ IV.

\subsection{Fe II UV $191(\lambda 1787)$}

Another striking similarity between the spectrum of Q0207-398 and those of Q03408-4505 and H0335-334 is the narrow emission line at $1787 \AA$ attributable to Fe II multiplet UV 191 ( $\lambda \lambda 1785.3,1786.7$, and 1788.0). These are among a group of only about a dozen QSOs in which we have seen this multiplet as a clear feature. Graham et al. (1995) discuss the spectrum of another QSO which, like that of Q0207-39, has quite narrow emission lines from $\mathrm{Fe}$ II UV 191, Al III, and Fe III UV 34, and no broad absorption lines. However, unlike Q0207 - 398, this new object also has strong $\mathrm{Fe}$ II emission in the 2200-2700 $\AA$ wavelength range. It probably has its origin in selective excitation; the other UV emission lines of Fe II are clearly not emitted in such narrow lines, although those of $\mathrm{Al}$ III are. Considerable additional searching of the multiplet tables, and comparisons to spectra of novae and other emission-line objects, does not yield an alternate identification. The origin of UV 191 is discussed further in Appendix C.

\subsection{Fe III UV 48 ( 22071$)$}

From Figure 2 it is clear that Fe III multiplet UV 48 is a good identification for the feature near $\lambda 2071$ in the bottom three spectra, while it is weak or absent in the upper four spectra. Note that a cosmic ray has been removed from the spectrum of $\mathrm{H} 0335-334$ at the position of the longest wavelength component of UV 48 , so the poor fit to the predicted intensity ratios within the multiplet is not surprising. The detailed fit is good in the cases of Q03408-4505 and $\mathrm{Q} 0207-398$. In the spectrum of Q1451 + 1017, there is a weak feature slightly shortward of the expected wavelength; because of the poor wavelength fit, we believe this to be a different, unidentified transition. The upper level of UV 48 is connected directly to the $\mathrm{Fe}$ III ground state through multiplet UV 1 at $1122 \AA$. Thus, although its upper level is at $11 \mathrm{eV}$, UV 48 can easily be pumped by continuum radiation longward of the Lyman limit, and therefore it should be a strong emitter from any $\mathrm{Fe}^{++}$zone which has at least moderate optical depth in UV 1. This sets it apart from UV 34 .

\subsection{He II $\lambda 1640$}

A final systematic trend visible in Figure 2 is that the top three spectra (those with weak Al III) all have quite noticeable He II $\lambda 1640$ emission, while in the bottom four spectra (the ones with strong $\mathrm{Al}$ III) the $\mathrm{He}$ II line is weak or cannot be detected, with an upper limit which shows that the $\mathrm{He}$ II/C IV intensity ratio is considerably lower than normal.

Paper II shows that, given the strong N v 1240 emission from most of these QSOs (Q1451 + 1017 being the conspicuous exception), this can only be an abundance effect. The helium line becomes weaker at high abundances because many of the helium-ionizing photons are absorbed 
by heavy elements rather than by helium. The $N \vee \lambda 1240$ / He II $\lambda 1640$ ratio is an especially sensitive measure of this because both lines come from the same ionization zone, and because $\mathrm{N}$ is a secondary element in stellar evolutionary processes and is therefore built up much more rapidly as a function of metallicity than most other heavy elements (see Paper II).

\subsection{III $\lambda 977$}

Relative to C IV $\lambda 1549$, the C III $\lambda 977$ line is clearly stronger in Q0207-398 than in any of the other QSOs. The upper limits for the other objects are 2-5 times smaller than the estimated strength in Q0207-398.

The C III $\lambda 977 / C$ III $] \lambda 1909$ intensity ratio has been proposed as a density indicator in BELR spectra (see Osterbrock 1991), and it is certainly true that strong $\lambda 977$ indicates that high-density gas is present. However, if a range of densities is present, the two lines will be produced in quite different components of the BELR. Strong $\lambda 977$ would be produced only in high-density gas subjected to high ionizing flux levels, while $\lambda 1909$ would come from lower density gas receiving lower ionizing flux, and the final intensity ratio would mostly measure the relative masses of the two components. We note also that some part of this emission is attributable to Ly $\gamma$ 2973; however, C III is definitely present (see Fig. 3).

\section{4. $\mathrm{Q} 0207-398$}

The key object in this study is Q0207-398. Its combination of narrow emission lines and profile differences gives us unusually good leverage for identifying which lines come from which BELR component. We describe first in considerable detail how we have deconvolved the various emission features into what we think is a "best guess" set of line strengths for each component, and then we discuss in turn our model for each component.

\subsection{Separation of Individual Profile Components}

The simplest description of the emission lines in this QSO is that they form three different groups. The process of disentangling these components starts in Figure $5 a$, where we overplot the observed profiles of Ly $\alpha, C$ IV $\lambda 1549$, and the Fe II UV 191 feature. C IV is obviously much broader and less symmetric than UV 191, so at least two profiles are needed.

UV 191 is a blend of three lines at $1785.27,1786.75$, and $1788.00 \AA$ which, for the physical conditions derived below, should all be of equal intensity. Experiments combining narrow Gaussian profiles at these wavelengths show that individual multiplet members with FWHM $\sim 900 \mathrm{~km} \mathrm{~s}^{-1}$ will combine together to give a sharply peaked line with $1000 \mathrm{~km} \mathrm{~s}^{-1} \mathrm{FWHM}$, as is observed. In view of the very modest broadening due to blending and the large errors which would arise from trying to disentangle it, we have used the entire UV 191 profile as our template for the narrowest emission component. Its wavelength was checked by fitting it to the Al III $\lambda \lambda 1854.72,1862.78$ doublet, which gives a good fit using the expected blended wavelength of $1786.7 \AA$

The $\mathrm{C}$ IV line is a blend of the two members of the $\mathrm{C}$ IV $\lambda \lambda 1548.20,1550.77$ doublet, which we expect to have a $1: 1$ (optically thick) intensity ratio for conditions within the broad emission-line region. To recover an unblended $\lambda 1548.20$ profile, we repeatedly shifted our best-estimate

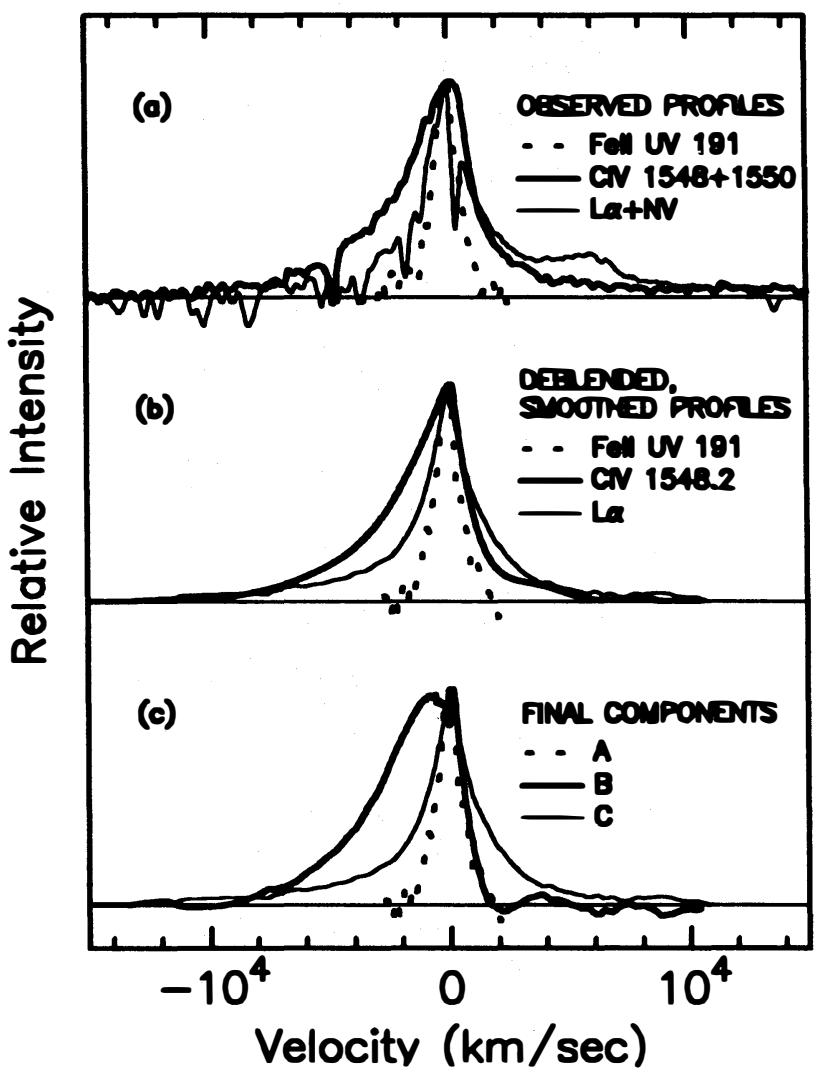

Fig. 5.-Q0207-398 line profiles. (a) Observed profiles of Fe II UV 191, C IV $\lambda 1549$, and the Ly $\alpha \mathrm{N}$ v blend. (b) The same data after extracting the $C$ IV $\lambda 1548.2$ profile from the blended doublet and removing $N v$ from the wing of $\mathrm{Ly} \alpha$ as explained in $\S$ 4.1. (c) The final components after subtracting the component $C$ contribution from the $C$ IV $\lambda 1548.2$ profile as explained in $\S 4.1$.

$\lambda 1548.20$ profile (initially the full blend) to $1550.77 \AA$ and scaled it to have half the total flux in the blend. Subtracting this from the blended profile gave us a new best estimate of the $\lambda 1548.20$ profile. In two iterations, this converged to a template from which we can very accurately model the full blended profile. The final $\lambda 1548.20$ profile is shown as the heavy line in Figure $5 b$; it has an extended blue tail but cuts off sharply on its red side at about the same velocity as the peak of the Fe II UV 191 line (dotted line in Fig. 5b).

Then we moved on to the observed Ly $\alpha, N$ v 1240 blend. $\mathrm{N} \mathrm{v}$ is another doublet which we expect to be near its optically thick $1: 1$ intensity ratio. For any reasonable shape for the red wing of $L y \alpha$, the $\mathrm{N} v$ line must be cutting off sharply on its red side in the same way as C IV 21548.20 . Therefore, we used the $\lambda 1548.20$ profile to fit the $\mathrm{N} \mathrm{V}$ $\lambda \lambda 1238.81,1242.80$ blend and subtracted it from the $L y \alpha$, $\mathrm{N} v$ blend. The intensity of $\mathrm{N} v$ was varied until we could see no residual features on the Ly $\alpha$ wing that obviously correlated with the shape of the $\mathbf{N} \mathbf{v}$ blend. After interpolating over many obvious absorption lines and smoothing, this left us with a Ly $\alpha$ profile which we used as the third of our three initial profiles; it is the moderately broad and very symmetric one shown as the light solid line in Figure $5 b$.

Up to this point, we consider the separation of these components to have been rather straightforward. But even sticking to the simplified idea of describing an entire unruly BELR with only three components corresponding to three physical regions, there is no reason to believe that lines such 
as $\operatorname{Ly} \alpha$ and C IV are emitted by only one of those three components. Although it is clear from Figure $5 \mathrm{~b}$ that no Fe II UV 191 comes from gas at the velocities characterizing the wings of the other two lines, there could be a fair amount of emission from the UV 191-forming region contributing to the central spike in the Ly $\alpha$ profile or to the area near the $C$ IV $\lambda 1548.20$ peak. The maximum contributions which do not produce holes in the residuals are $22 \%$ of $L y \alpha$ and $24 \%$ of $\lambda 1548.20$. Similarly, some of the C IV emission could be produced by gas having the same profile as $L y \alpha$, accounting for up to $51 \%$ of the $C$ IV flux.

To arrive at a "best guess" of the actual contributions of each region to each profile, we have combined the basic profile information given above with the intensity ratios between different lines predicted by the CLOUDY photoionization calculations described below (see also Appendix B). The strengths of the individual emission lines were measured by fitting combinations of these templates to each observed blend, and when ambiguous, the amount of each component which contributed to these fits was guided by photoionization calculations.

Specifically, according to our models there is no way to produce the $\mathrm{Ly} \alpha: \mathrm{N} \mathrm{v}: \mathrm{C}$ IV:Al III intensity ratios that would come from assigning the maximum possible UV 191 profile contribution to the $L y \alpha$ and C IV $\lambda 1548.20$ profiles, so we discard that possibility. The contribution cannot be more than about $25 \%$ of the maximum, or $5 \%-6 \%$ of the total flux in each of the $\mathbf{L y} \alpha, \mathbf{N} \mathrm{v}$, and $\mathrm{C}$ Iv lines. The models also show that it is extremely hard to produce C III] $\lambda 1909$ without producing C IV $\lambda 1549$. Since $C$ III] will turn out to have roughly the same profile as $L y \alpha$, we subtract the maximum possible Ly $\alpha$ profile contribution $(51 \%)$ from the $\lambda 1548.20$ profile. This defines a new profile which has little flux to the red of the line peak, but a long tail to the blue.

Our final adopted profiles are shown in Figure 5c. They are as follows:

Component A.- These are the narrowest lines. They are symmetric, with FWHM $=1000 \mathrm{~km} \mathrm{~s}^{-1}$ and FWZI $=3500$ $\mathrm{km} \mathrm{s}^{-1}$. They are typified by the $\mathrm{Al}$ III $\lambda 1857$ doublet and $\mathrm{Fe}$ II UV 191, but they also seem to include C II, C III, and $\mathrm{Si}$ II features and probably also the Fe III multiplet (UV 34) around $\lambda 1909$ (see $\S 3$ ).

Component B.-This group includes C IV $\lambda 1549, \mathbf{N}$ v $\lambda 1240$, and $O$ VI $\lambda 1034$. These are the broadest lines $\left(\mathrm{FWHM}=3900 \mathrm{~km} \mathrm{~s}^{-1}\right)$, and they have a pronounced blueward asymmetry (with the blue tail going out to -9000 $\mathrm{km} \mathrm{s}^{-1}$ ).

Component C.-This final set of lines includes Ly $\alpha, \mathrm{Si}$ IV 21397, and C III] 21909 . These have FWHM $=1900 \mathrm{~km}$ $\mathrm{s}^{-1}$, with moderately symmetric cores and extended wings reaching zero intensity at about $\pm 11,000-12,000 \mathrm{~km} \mathrm{~s}^{-1}$. There is no unblended line with this profile; component C's existence is inferred because several of the observed blended profiles cannot be produced by any combination of just components $\mathrm{A}$ and $\mathrm{B}$.

We describe these three components in turn, with the emphasis on the physical properties of component A. Component $B$ is the subject of Paper II.

\subsection{Component A: Very High Density Gas}

Here we show that the emitting region of the narrowest emission lines (component A), Al III, Fe II UV 191, and probably $\mathrm{Si}$ II, $\mathrm{O}$ I, is one of very high gas densities.
4.2.1. $\mathrm{Al}$ III $\lambda 1857$

$\mathrm{Al}$ III $\lambda 1857$ is the only line which we can uniquely identify with component $A$ and which is incorporated in the spectral synthesis code. CLOUDY does not make predictions for the $\mathrm{Fe}$ II and $\mathrm{Fe}$ III lines because of the great complexity of those ions. The basic goal of the modeling effort for component $\mathrm{A}$, therefore, was to produce a sufficient equivalent width of Al III $\lambda 1857$ without overproducing any of the other lines.

\subsubsection{Observational Constraints on the Density}

Si III] $\lambda 1892$ appears to be weak or absent in component A. This is important, since we will show in the next section that the small $\mathrm{Si}$ III]/Al III intensity ratio will constrain component A to have very high densities. Since $\mathrm{Si}$ III] would fall quite near a prominent shoulder which is observed on the blue wing of $\mathrm{C} \mathrm{III]} \lambda 1909$, it is important to illustrate why we do not think this line can be at all strong. Figure $6 a$ shows the best fit that we can get to the $\lambda 1909$ blend if we assume that only Si III] and C III] are present. We tried many combinations of components $\mathrm{A}, \mathrm{B}$, and $\mathrm{C}$, but this particular fit uses only component $\mathrm{C}$. The fit is unacceptable because the observed peaks fall significantly to the red of the fitted peaks. Component A profiles give an even worse fit to the data because of the narrowness of the predicted lines.

Figure $6 b$ shows a much better fit to the observed profile. In this case the blend was modeled by a combination of $\mathrm{C}$ III] and Fe III multiplet UV 34. The individual lines in the $\mathrm{Fe}$ III multiplet were modeled with component A profiles

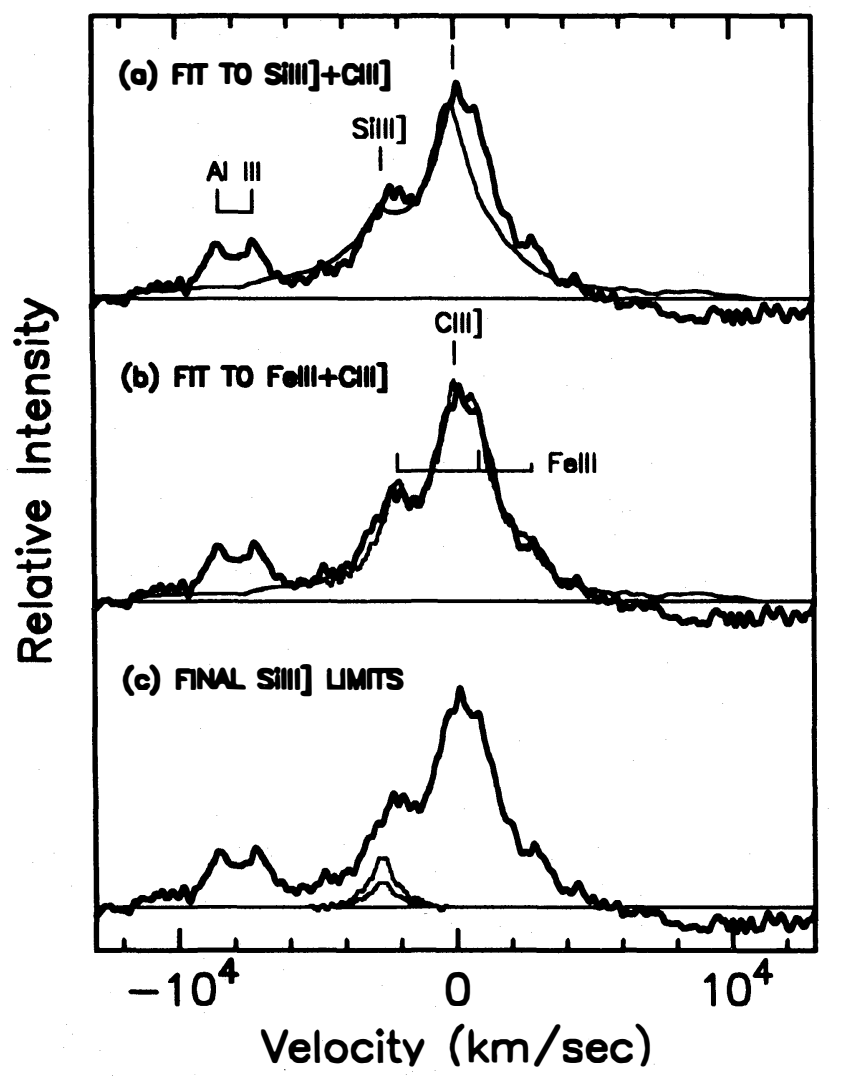

Fig. 6.-(a) The best-fit that we can get to the $\lambda 1909$ blend in Q0207-398 if we assume that only $\mathrm{Si}$ III] and C III] are present. (b) An alternate fit which assumes that the blend is instead made up of only Fe III multiplet UV 34 and C III]. (c) Final upper limits to the $\mathrm{Si}$ III] strength (see $\S 4.2 .2$ ). 
having the same relative intensities as in H0335-398 (see Appendix A), since we regard the latter QSO as having an essentially pure component A-type spectrum (however, component $\mathrm{C}$ profiles give an equally good fit). A component C profile is required to get a good fit to C III] $\lambda 1909$; the component A profile is too narrow. Once the profiles have been chosen, this model has only one free parameter (the $\mathrm{Fe}$ III/C III] intensity ratio), yet it gives a very good fit. We conclude that $\mathrm{Fe}$ III and $\mathrm{C}$ III] are the main contributors to the $\lambda 1909$ blend.

This implies that $\mathrm{Si}$ III] must be fairly weak. We have considered the two different upper limits on the $\mathrm{Si}$ III] strength shown in Figure $6 c$. These are both component A profiles at the position of Si III]. The higher limit ignores the Fe III fit and requires only that the residual not have a noticeable hole at the position of $\mathrm{Si}$ III]; it gives $\mathrm{Al}$ III/ $\mathrm{Si}$ III] $>1.7$. We regard this as the smallest ratio which is at all reasonable. But when we include Fe III in the fit, as we believe we must, the limit increases to $\mathrm{Al}$ III/Si III] $\gtrsim 3.3$, as shown by the smaller Si III] limit in Figure $6 c$. The paucity of $\mathrm{Si}$ III] in component $\mathrm{A}$ is important, as its critical density is among the highest for the ultraviolet intercombination lines (see $\S 3.2$ ), and thus it will set the highest lower limits to the hydrogen particle density.

\subsubsection{Photoionization Calculations}

We generated grids of models using the ionizing continuum described in Appendix B and shown in Figure 5 of Paper II. The basic approach is to run grids of several hundred models covering a wide range of different gas densities $n_{\mathrm{H}}$ and hydrogen ionizing photon fluxes $\Phi(\mathrm{H})$. Contour plots are then made of the intensity ratios and equivalent widths of all the predicted emission lines falling in the observed wavelength range. By tracing the contours corresponding to observed values or limits onto a master plot of $n_{\mathrm{H}}$ versus $\Phi(\mathrm{H})$, it is possible to delimit the range of parameters which could produce the observed spectrum. This is analogous to the classical methods for analyzing $\mathrm{H}$ II region spectra which are described in Seaton (1960), Osterbrock (1989), and elsewhere.

Figure $7 a$ is a contour plot showing the predicted equivalent width of $\mathrm{Al}$ III $\lambda 1857$ per unit covering factor. The observed $W_{\lambda}(\mathrm{Al}$ III $)$ is $1.8 \AA$. The covering factor is unknown, but clearly it must be less than 1.0 since components $B$ and $\mathrm{C}$ also must receive an appreciable amount of ionizing flux. We arbitrarily chose $50 \%$ covering factor as a representative maximum value to use in constraining the physical conditions, which means that the values on Figure $7 a$ should be compared to an observed lower limit of 1.8 $\AA / 0.5=3.6 \AA$ (0.6 dex).

Figure $7 b$ shows the limits that come from the $\mathrm{Al}$ III/Si III] ratio, estimated above. Moving from left to right, the solid contours represent ratios of $0.1,1.0,10.0$, etc.

The intersection of Figures $7 a$ and $7 b$ produces a region of acceptability on the density-flux plane, based upon the $W_{\lambda}(\mathrm{Al}$ III $)$ and the $\mathrm{Al}$ III/Si III] ratio. The result is shown in Figure 8. The solid limiting contour (labeled " 1 ") marks the requirement that the gas covers less than half of the continuum source $\left[W_{\lambda}(\mathrm{Al}\right.$ III $\left.)>3.6 \AA\right]$; this condition is met inside this contour. In the previous section, a conservative lower limit to the $\mathrm{Al}$ III/Si III] ratio was found: $>1.7$. In Figure 8 we show this limiting contour as a dashed line (labeled " 2 "); the condition is met for values of density and flux lying to the right of this line. Considering both limiting contours restricts the hydrogen particle density to be larger than about $10^{12.3} \mathrm{~cm}^{-3}$ (or larger if the limiting $\mathrm{Al} \mathrm{III/Si} \mathrm{III]}$ ratio of 3.4 is used). At these densities, most of the normally strong UV emission lines observed in QSOs are at or near their thermalization limits.

Another constraint on the allowed parameter space in Figure 8 comes from the lower limit on the $\mathrm{Al}$ III/Ly $\alpha$ ratio (0.05 or -1.3 dex; see Table 4$)$ for component $A$. This indicates a region on the density-flux plane which nearly coincides with the intersection of Figures $7 a$ and $7 b$ just described. Values of this ratio must lie within the oval shaped dot-dashed contour (" 3 ") in Figure 8. Combining these limits with those above decreases somewhat the allowed area on the density-flux plane. The limiting contour of the $\mathrm{Ly} \alpha / \mathrm{Al}$ III ratio as well as the $W_{\lambda}(\mathrm{Al}$ III) places an upper limit to the density, $n_{\mathrm{H}}$, of $\sim 10^{13.4} \mathrm{~cm}^{-3}$. Tests show that these limits on the density and flux are not strongly dependent on continuum shape or metallicity.

Taken together, the constraints mentioned thus far allow a range of 1.5 dex in values of the ionization parameter, $U(\mathrm{H}) \equiv \Phi(\mathrm{H}) / n_{\mathrm{H}} c$, centered near a value of $U(\mathrm{H}) \sim 0.01$. The ratio of $\mathrm{Al}$ III to a higher ionization line further limits the ionization parameter, but with the caveat that there is some dependence on the continuum shape. The observational limit on the $\mathrm{Al}$ III/C IV ratio restricts values of density and flux to lie below the dotted line (" 4 ") on Figure 8. A more constraining ratio is the $\mathrm{Al}$ III/Si IV ratio limit, shown as a triple-dot-dashed line (" 5 "); values must lie below this line. The range of allowed values of $U(\mathrm{H})$ is reduced to 0.75 dex. The remaining allowed area on the density - flux plane in Figure 8 is bounded by the $\mathrm{Al} \mathrm{III/Si} \mathrm{IV} \mathrm{ratio} \mathrm{at} \mathrm{high} \mathrm{fluxes,}$ by the $\mathrm{Al}$ III/Ly $\alpha$ ratio at low fluxes, by the $\mathrm{Al} \mathrm{III} / \mathrm{Si}$ III] ratio at low densities, and by $W_{\lambda}(\mathrm{Al}$ III) (among others) at high densities.

The final limits from Figure 8 are $10^{12.2}<n_{\mathrm{H}}<10^{13.3}$ $\mathrm{cm}^{-3}$ and $10^{20.1}<\Phi(\mathrm{H})<10^{21.2} \mathrm{~cm}^{-2}$. We adopt the midpoints of these ranges, $n_{\mathrm{H}}=10^{12.7} \mathrm{~cm}^{-3}$ and $\Phi(H)=10^{20.65} \mathrm{~cm}^{-2}$, as representative of the conditions in component $\mathrm{A}$. This is the same flux in hydrogen ionizing photons as is favored to be incident upon component $\mathbf{B}$ $\left[\Phi(H) \approx 10^{20.7} \mathrm{~cm}^{-2}\right.$; Paper II $]$. These parameters imply a covering factor of $\sim 0.28$. For the adopted continuum shape and a specific luminosity at $1216 \AA$ of $\lambda L_{\lambda}=3.67$ $\times 10^{46}\left(100 / H_{0}\right)^{2}$ ergs s$^{-1}$ (assuming $q_{0}=0.5$ and isotropic emission), this value of $\Phi(\mathrm{H})$ corresponds to a radial distance between the ionized gas and the continuum source of $r \sim 5 \times 10^{17}\left(100 / H_{0}\right) \mathrm{cm}$. In Table 5, we show the predicted line intensities of component $\mathrm{A}$ for the chosen values of $\boldsymbol{n}_{\mathrm{H}}$ and $\Phi(H)$. The predictions are normalized to the observed flux in the Al III emission line.

The observed $\mathrm{Al}$ III/He II $\lambda 1640 \geq 1.67$, while the ratio calculated from the models does not reach that value anywhere on the density-flux plane (Fig. 7c) and is in the range 0.4-0.6 within the region constrained by the other line ratios. A softer big bump cutoff energy for the same value in $\alpha_{o x}$ would help, as would higher metallicities. For example, a big bump with a cutoff energy of $18 \mathrm{eV}$, the minimum allowed by the curvature of the observed UV continuum (Paper II) and which happens to be the ionization potential of $\mathrm{Al}^{+}$, would produce an $\mathrm{Al}$ III/He II ratio of about 1 while altering the other results little. Increasing the metallicity to $10 Z_{\odot}$ will produce the observed lower limit to the $\mathrm{Al}$ III/He II ratio in the region of interest on the density-flux plane while altering the other results little [except to increase the 


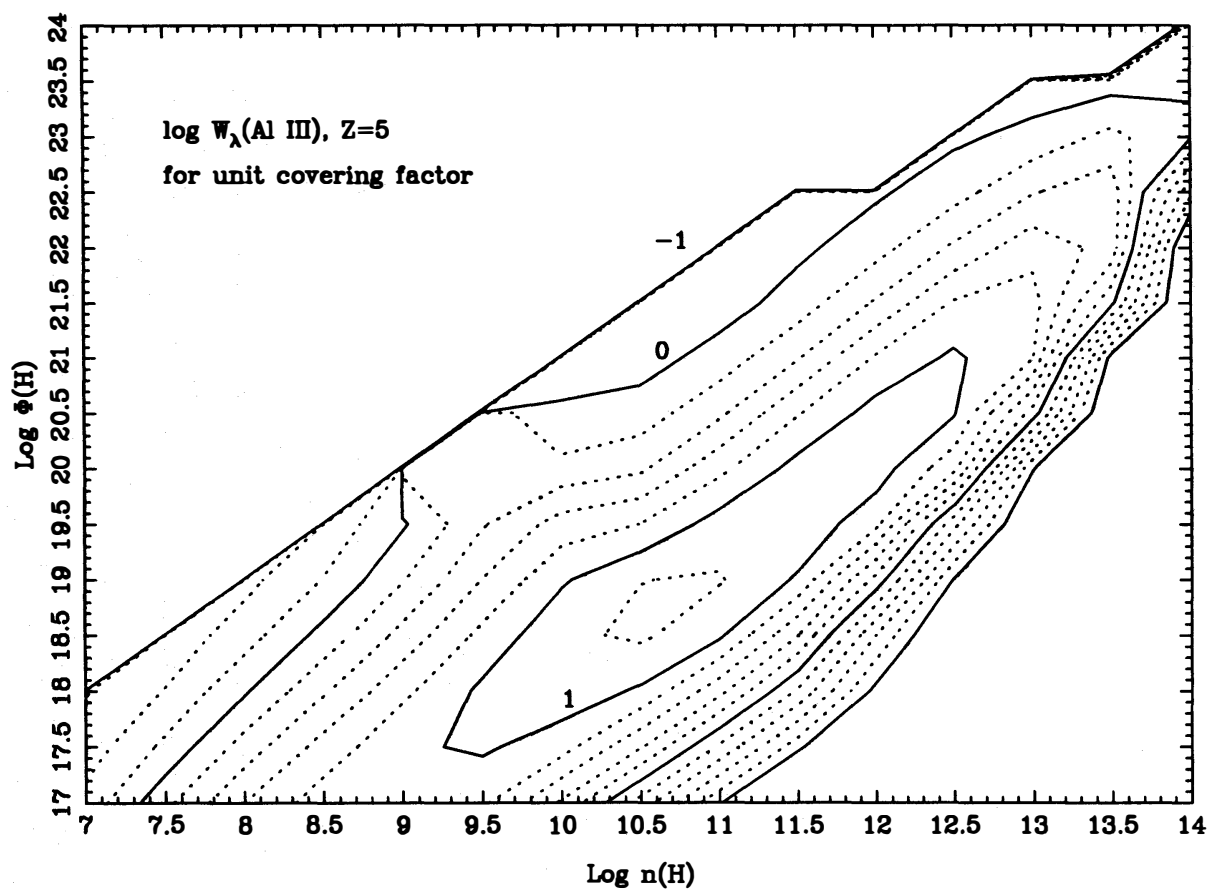

FIG. $7 a$

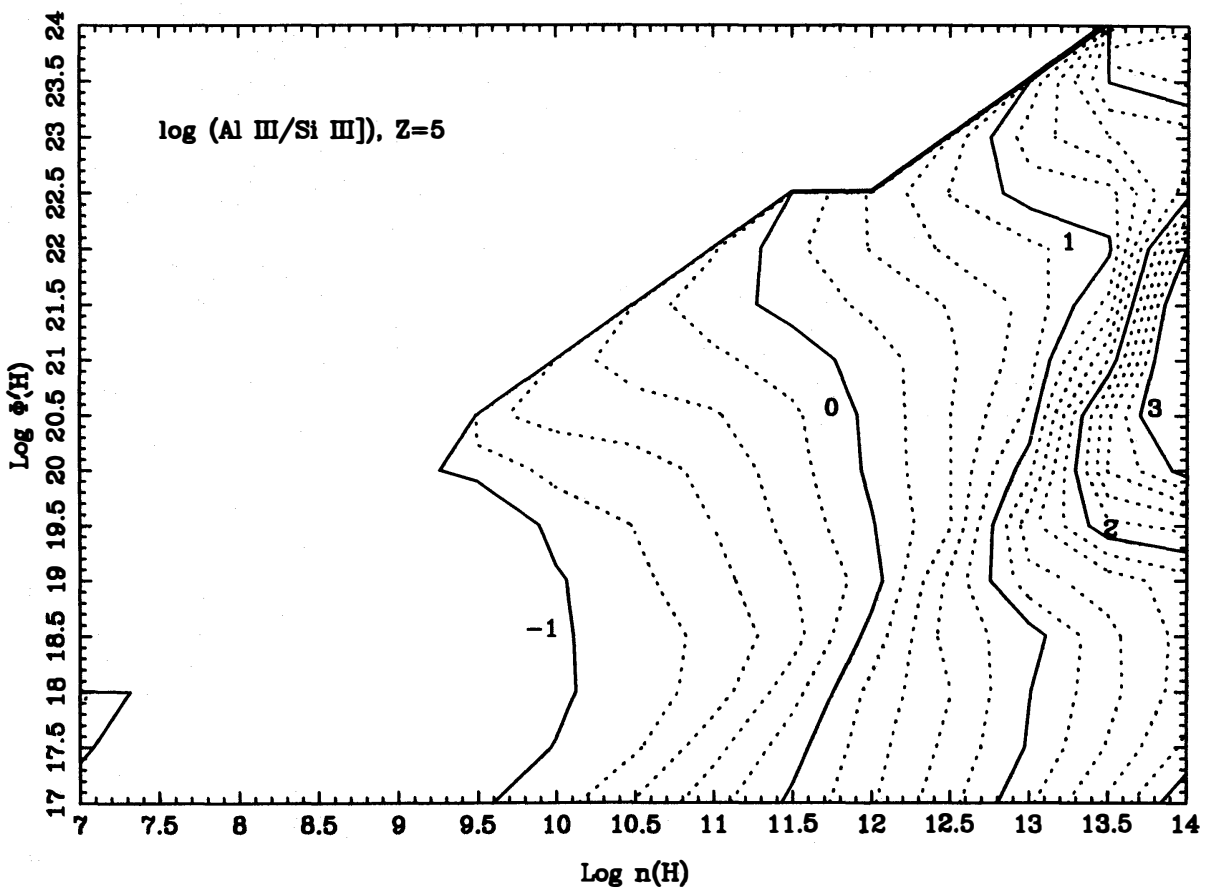

Fig. $7 b$

Fig. 7. - (a) Predicted contours of $\log W_{\lambda}(\mathrm{Al}$ III) for component A, as a function of density and flux of hydrogen ionizing photons, for a covering factor of 1.0 and for metallicity $Z=5 Z_{\odot}$. The solid contour in the center has a value of $1(10 \AA)$ and decreases outward in increments of 0.2 in the log. The observed equivalent width must be scaled by (covering factor) ${ }^{-1}$ before comparing to this figure; the observed $W_{\lambda}(\mathrm{Al}$ III) $=1.8 \AA$ then scales to log $(3.6)=0.6$ dex for the ass umed covering factor of 0.5 . (b) Same as $(a)$ for $\log \left(\mathrm{Al} \mathrm{III} / \mathrm{Si}\right.$ III]). This ratio is mainly a function of $n_{\mathrm{H}}$. The leftmost solid contour has value 0 , with values increasing to the right in increments of 0.2 in the log. The observed limits $[\log (\mathrm{Al} \mathrm{III/Si} \mathrm{III]})>0.22$ in the most relaxed case; see $\S 4.2 .2]$ indicate densities larger than $\sim 10^{12} \mathrm{~cm}^{-3}$. (c) Same as for $\log$ (Al III/He II). The central solid contour has value of 0 in the log, with values decreasing outward in increments of 0.2 in the log. The observed limit to the ratio is $\gtrsim 0.2$ in the log. These calculations fail to reprod uce that limit anywhere; see $\S 4.2 .3$ for details.

$W_{\lambda}(\mathrm{Al}$ III) by a factor of $\sim 2]$. In Paper II we emphasize that $Z=5 Z_{\odot}$ is the minimum metallicity required by the highionization line ratios in component $\mathrm{B}$, and it might well be larger. Doubling the metallicity is therefore a perfectly acceptable solution. However, given the uncertainties in the atomic physics for third row elements, uncertainties in the elemental abundances as a function of metallicity, and a desire to keep the number of free parameters at a minimum, we choose not to fine-tune either the continuum shape or the metallicity.

There is also an important discrepancy between the predicted and observed Si II intensities. This is probably the signature of a pumping mechanism, as is discussed in Appendix C. The other large difference between the obser- 


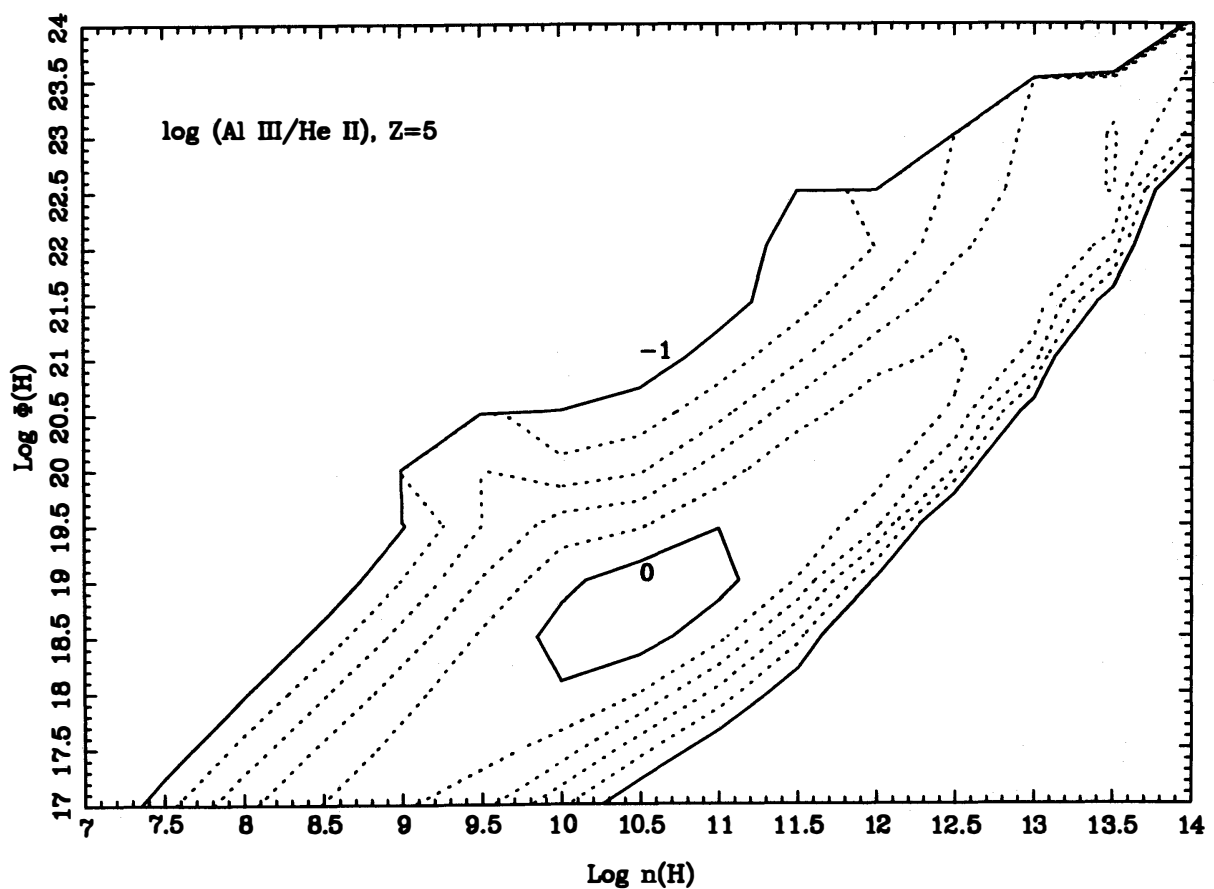

FIG. $7 c$

vations and the model is that the $O \mathrm{III}] \lambda 1665$ intensity is underpredicted by a factor of 10 . We do not see any way to make this intercombination line in such high-density gas, and we can only suspect that we have either misidentified the line or attributed it to the wrong component.

\subsection{Component B: Outward-Flowing, High-Ionization Gas}

Paper II explores simulations of this component. The approach there is to find the ionizing continuum shape and other input parameters that will produce the observed intensity ratio $\mathrm{N} v \lambda 1240 / \mathrm{He}$ II $\lambda 1640>10$ (and be consis- tent with all other measured values) with the least possible abundance enrichment. This requires at least 5 times solar metallicity. The most important constraints come from the measured limit of $\mathrm{N} \mathrm{v} / \mathrm{He}$ II and the measured value of $W_{\lambda}(\mathbf{N}$ v), and from the measured $\mathrm{N} v / C$ IV and $\mathrm{N} v / \mathrm{O}$ vI ratios. These indicate $n_{\mathrm{H}} \sim 10^{11} \mathrm{~cm}^{-3}$ and $\Phi(\mathrm{H}) \sim 10^{20.7}$ $\mathrm{cm}^{-2} \mathrm{~s}^{-1}$. Since we find similar values of $\Phi(\mathrm{H})$ for components $\mathrm{A}$ and $\mathrm{B}$, they are at similar distances from the nucleus unless the two components see different continua.

The extended blueward tail on the profile of component B (Fig. 5c) strongly suggests a gas flow. Such a flow must

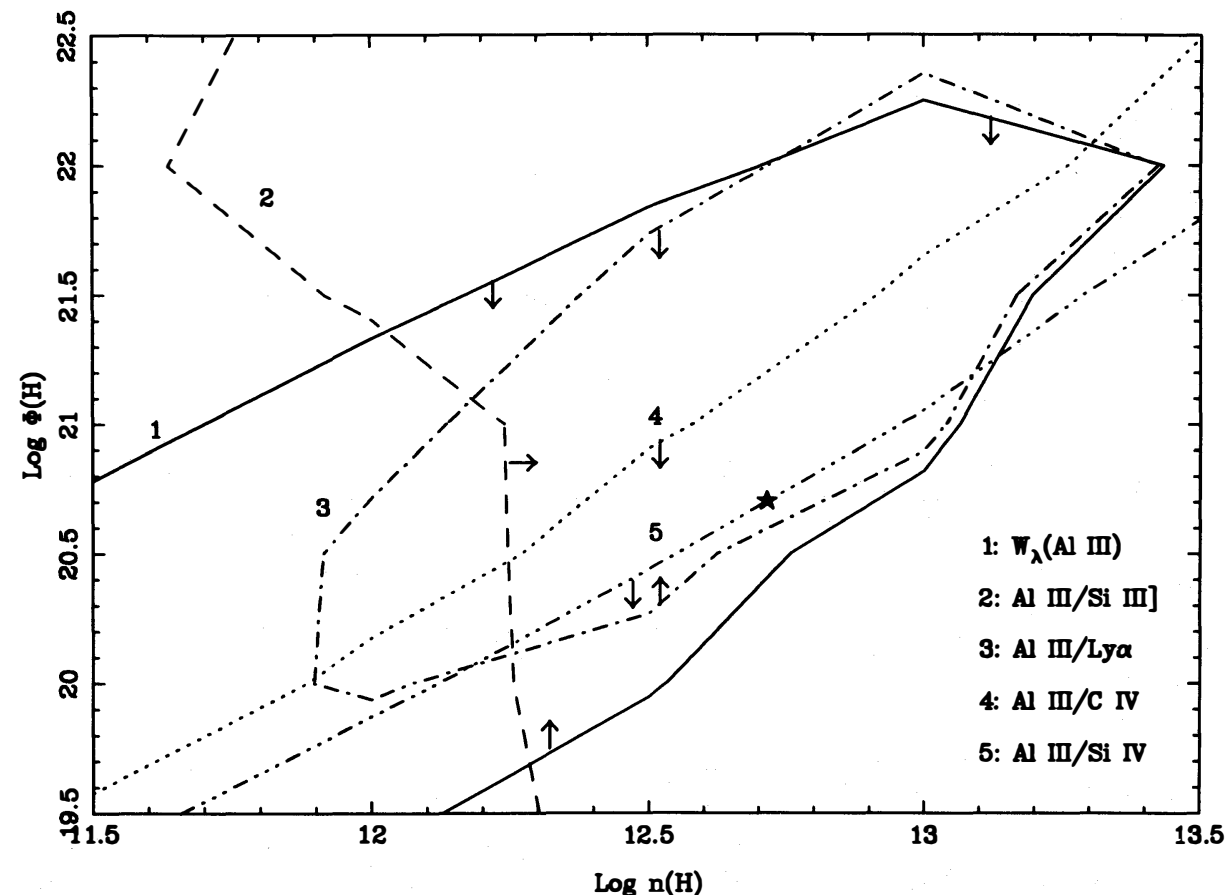

FIG. 8.-All available limits (see Table 4) for component A, as a function of density and flux of hydrogen ionizing photons. Values must lie inside contour 1 , to the right of contour 2 , inside contour 3 , beneath contour 4 , and beneath contour 5. The star marks the adopted $\Phi(\mathrm{H}), n(\mathrm{H})$ values from $\S 4.2 .3$. 
TABLE 5

\begin{tabular}{|c|c|c|}
\hline \multicolumn{3}{|c|}{ EMISSION LINE STRENGTHS ${ }^{a}$} \\
\hline Line & Measured & Predicted \\
\hline Ly $\gamma 973 \ldots \ldots \ldots$ & $\leq 2.2$ & 0.4 \\
\hline $\mathrm{C}$ III $977 \ldots \ldots \ldots$. & $\leq 2.8$ & 0.8 \\
\hline $\mathrm{N}$ III $991 \ldots \ldots \ldots$. & $<0.7$ & 2.0 \\
\hline Ly $\beta 1026 \ldots \ldots \ldots$ & $<3.8$ & 0.3 \\
\hline $\mathrm{O}$ vi $1034 \ldots \ldots \ldots$ & $\leq 5.9$ & 0.0 \\
\hline Ly $\alpha 1216 \ldots \ldots \ldots$ & $<40.8$ & 25.1 \\
\hline $\mathrm{N} v 1240 \ldots \ldots \ldots$ & $<9.6$ & 0.2 \\
\hline Si II $1263 \ldots \ldots \ldots$. & 4.6 & 0.1 \\
\hline O I $1303 \ldots \ldots \ldots$ & $<1.9$ & 1.0 \\
\hline Si II $1307 \ldots \ldots .$. & 4.0 & 0.0 \\
\hline C II $1335 \ldots \ldots \ldots$ & 1.4 & 0.6 \\
\hline Si IV $1397 \ldots \ldots .$. & $<2.9$ & 2.5 \\
\hline O Iv] $1402 \ldots \ldots$ & $<0.3$ & 0.1 \\
\hline $\mathrm{N}$ iv] $1486 \ldots \ldots$ & $<3.8$ & 0.0 \\
\hline C Iv $1549 \ldots \ldots \ldots$ & $<8.4$ & 4.9 \\
\hline He II $1640 \ldots \ldots$. & $<1.2$ & 3.9 \\
\hline O III] $1665 \ldots . .$. & 1.1 & 0.1 \\
\hline $\mathrm{N}$ III] $1750 \ldots \ldots$. & $<0.9$ & 0.0 \\
\hline Fe II $1787 \ldots \ldots \ldots$ & 1.9 & $\ldots$ \\
\hline Si II $1814 \ldots \ldots \ldots$. & 0.7 & 0.6 \\
\hline Al III $1857 \ldots \ldots$. & 1.8 & 1.8 \\
\hline Si III] $1892 \ldots \ldots$ & $<1.1$ & 0.5 \\
\hline C III] $1909 \ldots \ldots$. & $<2.7$ & 0.0 \\
\hline Fe III $1909 \ldots \ldots \ldots$ & 4.0 & $\ldots$ \\
\hline Fe III? $2071 \ldots \ldots$ & $<1.5$ & $\ldots$ \\
\hline $\left.\mathrm{N}_{\text {II }}\right] 2142 \ldots \ldots$. & $<0.4$ & 0.0 \\
\hline $\mathrm{C}$ II] $2336 \ldots \ldots$. & $<0.4$ & 0.0 \\
\hline
\end{tabular}

have a substantial velocity gradient, which will affect the transfer of otherwise saturated emission lines. Continuum fluorescence is included as an excitation mechanism for all lines in our calculations (see Ferguson, Ferland, \& Pradhan 1994). For thermal line widths, this is usually not a significant contribution, but fluorescence will be important if lines are significantly turbulently broadened, since they are then able to absorb more of the continuum. We computed a series of models in which turbulence was added to the line widths. As expected, the equivalent widths of lines did increase, by as much as a factor of 3 for a turbulent velocity equal to the observed line widths. The relative line intensities did not change by more than $50 \%$ for even the most extreme case, since all lines are strengthened by this process. We do not consider turbulence further here, since the main effect is only to lower the required gas covering factor by the amount the equivalent widths change.

\subsection{Component C: An Almost-Normal BELR?}

Finally we come to component $\mathrm{C}$, for which the template line is the more or less symmetric Ly $\alpha$ profile. This becomes the catch-all for whatever observed line flux cannot be explained by our models of components $\mathrm{A}$ and $\mathrm{B}$. It appears to produce most of C III] $\lambda 1909$ as well as almost all of the Ly $\alpha$ and Si IV $\lambda 1397$. Our "best-guess" separation of the line components attributes $51 \%$ of the $N$ v $\lambda 1240$ and C IV $\lambda 1549$ flux to component $C$.

Since the line strengths measured for this component $\mathrm{C}$ are basically residuals from fits to other components, we have not attempted to model this region, and therefore we cannot give quantitative limits on $n_{\mathrm{H}}$ or $\Phi$. However, Table 4 shows that except for Ly $\alpha$ being 2 times too strong, component C's spectrum is very similar to that of the two "normal" QSOs in our present sample (Q0000-398 and
Q2212 - 299) which have comparable luminosities. Those two objects in turn have $L y \alpha / C$ IV ratios about 2 times larger than in the composite QSO spectrum presented by Boyle (1990).

The other way in which the spectra of Q0000-398, Q2212-299, and all three emission-line components of Q0207-398 appear to be unusual is that $\mathrm{Si}$ III] $\lambda 1892$ is very weak relative to $\left.\mathrm{C}_{\mathrm{III}}\right] \lambda 1909$. The $\left.\mathrm{Si} \mathrm{III}\right] / \mathrm{C}$ III] ratio is $<0.15$ in these three QSOs, whereas it is typically about 0.3 in Seyfert galaxies and in four narrow-lined QSOs observed by Ulrich (1989), as well as in Q1451 + 1017 (Table 4).

We conclude that component $\mathrm{C}$ has a spectrum which is similar to those of two of the three "normal " narrow-lined QSOs in our sample, and which is much more like the spectra of generic quasars and Seyfert galaxies than like the spectra of components A or B.

\section{DISCUSSION}

\subsection{Bloated Stars?}

Component $\mathrm{A}$ is $>20$ times denser than component $\mathrm{B}$. It appears to have a covering factor $\Omega / 4 \pi$ and incident ionizing flux $\Phi(\mathrm{H})$ similar to component B, suggesting that the two components might be in the same location. Component A has an approximately Gaussian velocity distribution with a width (FWHM $\left.=1000 \mathrm{~km} \mathrm{~s}^{-1}\right)$ that is characteristic of velocities of stars deep within the nuclei of giant elliptical galaxies such as M87 (Sargent et al. 1978; Harms et al. 1994). Component B, on the other hand, has a velocity profile which is consistent with a gas flow, either inward or outward.

A natural interpretation of these facts is that component $A$ is made up of dense concentrations that are undergoing mass loss (whose origin is intrinsic, due to external heating, or both) to form component $B$. The gas densities inferred for component $\mathrm{A}$ are in fact quite close to the gas densities characteristic of the atmospheres of stars. We suggest that component A is in fact made up of "bloated" stars which are in the vicinity of the QSO nucleus (see Alexander \& Netzer 1994) and which are having gas driven off by radiation pressure from the nucleus.

Mathews (1983) estimated the conditions needed for radiatively driven mass loss from stars. The conditions he assumed are radically different from those we find here, or are currently thought to characterize quasars. Our clouds are much closer to the continuum source and so are exposed to a far stronger incident continuum. Our standard parameters for the Al III region correspond to Mathews's parameter $f_{\mathrm{uv}}=60$ rather than unity. Further, our continuum is far harder; the mean ionizing photon energy is roughly $40 \mathrm{eV}$ rather than $13.6 \mathrm{eV}$. Finally, the opacity of the gas is increased considerably by the enhanced metals. All these effects combine to make radiative acceleration more important than Mathews found.

Our simulations predict the radiative acceleration, and stellar mass loss will occur if this exceeds the star's surface gravity. Radiative acceleration arising from all continuous opacity sources and lines is calculated. This includes all stages of ionization of the first 30 elements and the 24,000 lines described in Appendix B. As expected, we find far more efficient acceleration than would be produced by continuous absorption alone. Figure 9 shows the predicted radiative acceleration at the cloud's illuminated face, on the density-flux plane. For the parameters adopted for the Al III 


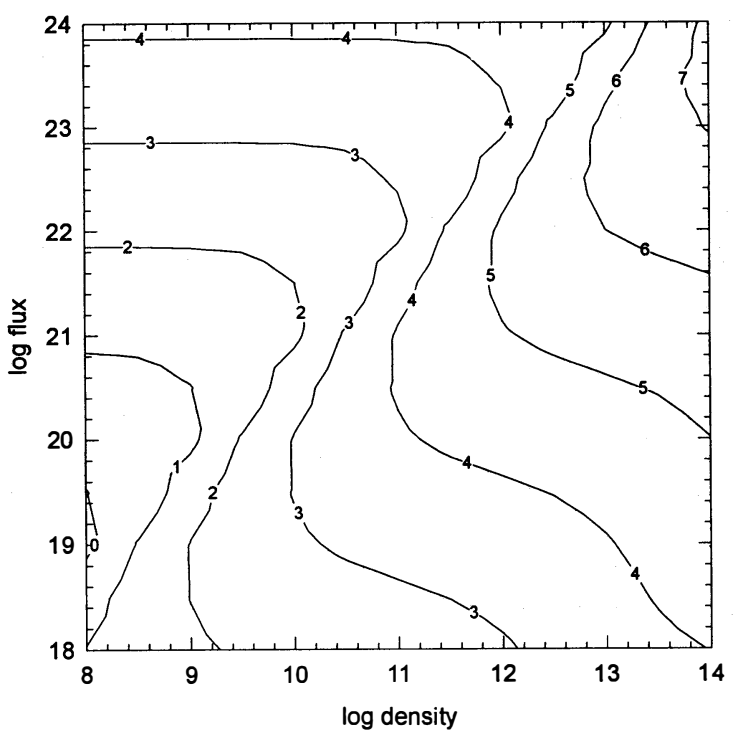

FIG. 9.-Predicted radiative acceleration for the case of $5 \times$ solar metallicity. The axes have the same meaning as in Fig. 7a. The contours show logarithmic (cgs) accelerations. Models calculated for solar metallicity depart only slightly from this figure except in the lower right corner, where they give results about 0.5 dex lower than are shown. For comparison, main-sequence stars have a surface gravity of about $10^{4} \mathrm{~cm} \mathrm{~s}^{-2}$.

region, the acceleration is $\sim 10^{5} \mathrm{~cm} \mathrm{~s}^{-2}$. This is about 4 times the surface gravity of the Sun, and thus it is clearly sufficient to drive a wind from giants and super giants, and from stars well down the main sequence.

The covering factor of component $\mathrm{A}(\Omega / 4 \pi \sim 0.2)$ seems rather large for stars alone, although this problem may be ameliorated if we do not observe the same continuum as the clouds. Thus, component A would be an expanded envelope of material that has not been fully accelerated away from the star. The accelerated gas is perceived as component $\mathbf{B}$. Similar scenarios for the BELRs of QSOs have been suggested by many investigators (Edwards 1980; Mathews 1983; Perry \& Dyson 1985; Penston 1988; Scoville \& Norman 1988; Voit \& Shull 1988; Kazanas 1989; Roos 1992; Alexander \& Netzer 1994), and Scoville \& Norman (1995) have put forth a similar idea for the origin of the BAL gas.

\subsection{Central Mass}

If component $A$ is made up of material in virialized orbits with random inclinations, then the central mass may be estimated from $M \approx v^{2} r / 2 G$, where $v$ is the full width at half-maximum intensity. Both the observed value of $v$ and our derived $r$ are considerably smaller than the numbers which are typically used in this type of estimate, so the corresponding central mass is surprisingly small: $3 \times 10^{7}$ $M_{\odot}$. Even using the most extreme allowed values in $\Phi(\mathrm{H})$ and $H_{0}$ predicts a mass of only a few times $10^{8} M_{\odot}$. This should be compared to the more typical estimates for luminous QSOs of $\sim 10^{9} M_{\odot}$.

It is possible that we have severely underestimated the radius because of beaming or obscuration, in a situation in which the ionized gas sees a very much higher continuum luminosity than we do from earth. Since the derived mass $\propto L_{\text {ion }}^{0.5}$, a factor of $10^{4}$ beaming is required to bring the mass into the usually estimated range. We cannot rule this out; in fact, there are serious discrepancies between the observed (possibly variable) optical to X-ray spectral index and the one needed to model component B adequately (Paper II).
Another obvious alternative to this result is to assume that component $\mathbf{A}$ is gas rotating in a disk. Since the line profiles are narrow and not obviously double-peaked, such a disk would probably be in a face-on orientation, making it impossible to estimate the central mass. In that case, the model in which component B is gas ablating off bloated stars would no longer explain the extended blue tails of the component B profile, since any radiative acceleration of gas driven off the component A material would be nearly in the plane of the sky.

\subsection{QSO Spectral Types}

The range of properties of the spectra shown in Figures 1 and 2 might be explained in the following way: the spectra near the top of the figures are of QSOs which have significantly smaller contributions from very dense regions than do the QSOs producing the spectra shown near the bottom of the figures. At the same time, there appears to be a progressive decrease, from top to bottom of the figures. At the same time, there appears to be a progressive decrease, from top to bottom in the figures, of the relative strength of the "normal" (component C) QSO spectrum.

This description of individual QSO spectra as the superposition of a few standard templates, but in changing proportions, is similar to the results of Francis et al. (1992), Wills et al. (1993), and Brotherton et al. (1994). Those papers dealt with the average properties of large samples and showed that BLR spectra can statistically be described as the superposition of a "very broad line region" (VBLR) and an "intermediate line region" (ILR). The VBLR discussed by Brotherton et al. is spectroscopically similar to component B in Q0207-398; it has similar line width (7000 $\mathrm{km} \mathrm{s}^{-1}$ ), it is blueshifted, and it can be fitted with the same ionization parameter (but they chose a somewhat higher density and therefore a correspondingly smaller $r$ ). Whether it has the same physical interpretation that we give to component $B$ is unclear.

Their ILR, however, is obviously quite different from component $\mathrm{A}$. It has a very different spectrum and is best modeled as relatively low-density gas $\left(n_{\mathrm{H}} \sim 10^{10} \mathrm{~cm}^{-3}\right)$ lying farther out $(r \sim 1 \mathrm{pc})$ than the VBLR gas. As described by Brotherton et al., the narrow-lined QSO Q1451+1017, which we include in this present study, is one of the prototypes for their ILR spectrum, and it is clear from our work here that it is quite different from component $\mathrm{A}$. In the case of Q0207-398, any ILR has presumably been included in our component $\mathrm{C}$.

The significance of the Brotherton et al. paper and its predecessors is that in general the BLR spectra of QSOs are better described by two components than by one. We show elsewhere (Baldwin et al. 1995) that a powerful generalized description of the BLR is as an ensemble of clouds covering a huge range both of gas densities and of distances from the continuum source, and that the distribution functions of those two parameters can be modeled statistically.

The case of Q0207-398 is different; in this particular QSO, the kinematical separation of the line profiles allows us to study some very well-defined, specific subregions within the BLR. It is unclear what fraction of all QSOs might have a region similar to component $A$ which does not happen to have a separable line profile. Since we identify Al III emission as an easily detectable signature of highdensity regions very close to the nucleus, we naively would have expected that strong $\mathrm{Al}$ III emission would be corre- 
lated with $\mathrm{C}$ IV profiles which indicate outflowing gas (either in emission or absorption). Various surveys of BAL QSO spectra do indicate that $\mathrm{Al}$ III is unusually strong in those objects (Hartig \& Baldwin 1986; Junkkarinen, Burbidge, \& Smith 1983; Weymann et al. 1991), although Weymann et al. (1991) would place the two BAL QSOs included in this study (H0335-336 and Q03408-4505) in a separate subclass of BAL QSOs distinguished by $\mathrm{Mg}$ II and $\mathrm{Al}$ III absorption troughs and also by particularly strong Al III and $\mathrm{Fe}$ III emission.

In Table 6 we list the total $\mathrm{Al}$ III equivalent widths for the objects observed here and also average values for some recent samples of BAL and non-BAL QSOs. Despite the remarkable overall similarities between the spectrum of Q0207-398 and those of our two BAL QSOs, $W_{\lambda}(\mathrm{Al}$ III) for Q0207-398 is smaller than the average for any of the QSO samples. In our models, $\mathrm{Al}$ III and most other emission lines from the high-density regions are completely saturated at the blackbody limit, and so it is plausible that the relatively small (factor of 4) differences in $W_{\lambda}(\mathrm{Al}$ III) shown in Table 6 are telling as much about the range in ionizing continuum shapes as about the amount of high-density gas present. The emergence of lines such as Fe II UV 191 or the strong $\mathrm{Si}$ II high-excitation lines may in the end be better indicators of the presence of large amounts of dense gas, for the reasons outlined in Appendix C.

Finally, we note that the striking similarities of the narrow emission-line characteristics indicate that a relationship between Q0207-398 and the two BAL QSOs described here (H0335 - 336 and Q03408 - 4505) may exist. The major difference between Q0207-398 and these two Mg II-type BAL QSOs is that the former has a strong component $B$ in emission, while the latter have many of the same lines in absorption from resonant scattering owing to outflowing material. We have suggested that component $B$ might also be associated with an outflowing wind. Might these two outflows have a common origin (e.g., acceleration of material from component A)? Could the existence of component B in either emission or absorption be caused by the orientation of the observer? If the two types of outflows are indeed of the same origin, then the scattered flux must (1) escape strongly preferentially in directions away from the viewing angle required to observe the BALs (otherwise we would observe a strong emission signature from the BAL gas in the two BAL QSOs, and it is clear that we do not), or (2) have a much smaller covering factor in the two

TABLE 6

Al III Equivalent WiDThS

\begin{tabular}{|c|c|}
\hline Object or Sample & $W_{\lambda}(\mathrm{Al} \mathrm{III})^{\mathbf{a}}$ \\
\hline 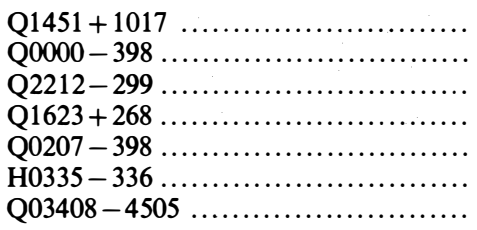 & $\begin{array}{r}<3.2 \\
2.1 \\
2.0 \\
3.5 \\
1.8 \\
7.6 \\
3.9\end{array}$ \\
\hline $\begin{array}{l}\text { Steidel \& Sargent } 1991 \text { non-BAL ...... } \\
\text { Weymann et al. } 1991 \text { non-BAL ......... } \\
\text { Weymann et al. } 1991 \text { all-BAL ........ } \\
\text { Weymann et al. } 1991 \mathrm{Mg} \text { II-BAL ...... }\end{array}$ & $\begin{array}{r}2-3^{\mathrm{b}} \\
4.0^{\mathrm{b}} \\
5.0^{\mathrm{b}} \\
5.6^{\mathrm{b}}\end{array}$ \\
\hline
\end{tabular}

a $\AA$ in rest frame.

b Average value for sample.
BAL QSOs, or (3) be preferentially destroyed in the two BAL QSOs. While current understanding concerning the BAL QSO phenomenon is that whether we observe BALs or not may depend on the observer's viewing angle to the QSO (Weymann et al. 1991), it is hard to understand how the emission properties of the scattering medium in the outflowing wind could be so extremely anisotropic (see Hamann, Korista, \& Morris 1993). The other two possibilities require differences to exist between the outflows.

\section{SUMMARY}

This study of seven $z \sim 2$ QSOs has produced the following main results:

1. The spectra show a range of properties which can be understood as a progressive difference in the relative contributions of different types of emission-line regions. At one extreme we see spectra which are a combination of lowionization lines including Al III $\lambda 1867, \mathrm{Fe}$ II UV 191, and various Fe III multiplets, and broad, asymmetric $N$ v $\lambda 1240$ and C IV $\lambda 1549$ lines. QSOs at the other end of the sequence emit the "classical" QSO spectrum including Ly $\alpha, C$ IV $\lambda 1549$, He II $\lambda 1640$, and C III] $\lambda 1909$.

2. Analysis of the spectrum of Q0207-398 shows the following:

(i) The spectrum of Q0207-398 is at an intermediate point in this sequence of properties. Its emission-line profiles come from three different gas components which we call A, B, and C. These components can be separated kinematically, permitting them to be studied individually.

(ii) Component A lines include Al III $\lambda 1857$, Fe II UV 191, and $\mathrm{Fe}$ III UV 34 and UV 48. They are produced in an extremely dense $\left(n_{\mathrm{H}} \gtrsim 10^{12.5} \mathrm{~cm}^{-3}\right)$ gas which, unless the ionizing radiation is not isotropic, lies $\sim 5 \times 10^{17} \mathrm{~cm}$ $(\sim 0.16 \mathrm{pc})$ from the ionizing continuum source. The emission-line profiles from this region are quite narrow, with FWHM $\approx 1000 \mathrm{~km} \mathrm{~s}^{-1}$.

(iii) Component B produces much of the $\mathrm{N} v$ and $\mathrm{C}$ IV flux in this same QSO. This gas is receiving about the same ionizing flux as component $\mathrm{A}$, but it is less dense $\left(n_{\mathrm{H}} \sim 10^{11}\right.$ $\mathrm{cm}^{-3}$ ). The line profiles have extended blue tails, which imply gas flowing at velocities $v \sim 10^{4} \mathrm{~km} \mathrm{~s}^{-1}$.

(iv) We suggest that component $A$ consists of the expanded envelopes of a large number of stars which are having their photospheres stripped away by radiation pressure from the QSO nucleus. Component B is the resulting outflowing wind. This model implies that the virial mass of the central object is only $10^{7}-10^{8} M_{\odot}$, if the ionizing continuum is isotropic.

(v) If component $\mathrm{A}$ is instead a rotating disk of some type, then the centrally peaked line profiles indicate that the disk probably is seen nearly face on. The above mass estimate would then be meaningless. But radiation pressure stripping of component $\mathrm{A}$ then could not account for the extended blue tail on the profile of component B, since the acceleration would be in the plane of the sky.

(vi) Q0207-398 also includes a third emission region, component $\mathrm{C}$, which we interpret as the spectrum which dominates in "normal" QSOs.

3. There are many similarities between Q0207-398 and the two Mg II BAL QSOs in our sample. We suggest that the low-ionization $\mathrm{Mg}$ II BAL phenomenon in general may be a consequence of stars being too close to the QSO 
nucleus, and that $\mathrm{Al}$ III $\lambda 1857$ emission is the indicator of those stars. The fraction of non-BAL QSOs which have significant amounts of outflowing gas can probably be determined from the statistics of $\mathrm{Al}$ III emission strength. Our sample includes several luminous QSOs which do not have detectable Al III emission, but several large and welldefined samples show that it is in fact very common.

We wish to thank the crew up on Cerro Tololo for their usual excellent assistance in obtaining these observations. We are grateful to the referee, Luc Binette, for a number of helpful suggestions. J. A. B. and G. J. F. thank STScI for support through grant GO 2306. G. J. F. and K. T. K. acknowledge the support of NSF (AST 93-19034) and NASA (NAGW-3315), and G. J. F. thanks the Cerro Tololo Inter-American Observatory for its hospitality. F. H. was supported by NASA grant NAG 5-1630, and B. J. W. was supported by NASA contract NAS 5-30934 (RSDC).

\section{APPENDIX A}

\section{DETAILS OF LINE STRENGTH MEASUREMENTS}

A1. Q1451+1017

The line profiles are composites of a broad $\left(F W H M=6200 \mathrm{~km} \mathrm{~s}^{-1}, \mathrm{FWZI}=25,000 \mathrm{~km} \mathrm{~s}^{-1}\right)$ base with an asymmetric red tail, and a strong narrow (FWHM $=850 \mathrm{~km} \mathrm{~s}^{-1}$ ) spike. The $\mathrm{C}$ Iv line has these two components in a broad-narrow intensity ratio of 4:1. The line strengths for Q1451+1017 in Table 4 are for a fit to each of the other lines of a profile with the two components in this intensity ratio, except that the integrated line intensity is given for Ly $\alpha$ and $\mathrm{Mg}$ II.

The narrow components offer an especially good opportunity for separating blends. Baldwin et al. (1988) had shown previously that the features near $1909 \AA$ are clearly C III] and Si III], rather than Fe III, but that C III] $\lambda 1909$ and $\mathrm{Mg}$ II $\lambda 2798$ are weaker relative to $L y \alpha$ and $C$ IV than in QSOs of more typical (broader) emission line widths.

Our improved data now show that the feature near $\lambda 1400$ is clearly Si IV $\lambda 1397$ rather than O IV] $\lambda 1402$ (the individual components are easily resolved), and they also allow us to measure $\mathrm{N} v \lambda 1240, \mathrm{O}_{\mathrm{I}} \lambda 1303, \mathrm{~N}$ IV] $\lambda 1486$, and $\mathrm{O}$ III] $\lambda 1665$. The fits to $\mathrm{C}$ IV $\lambda \lambda 1548.20,1550.77$ and Si IV $\lambda \lambda 1393.76,1402.77$ indicate that within each doublet the lines have a $1: 1$ intensity ratio as would be expected if the lines are optically thick, rather than the 2:1 value expected in the optically thin case.

The core of the Ly $\alpha$ emission line appears to extend to the red of the velocities of the other lines (see Fig. 3 of Baldwin et al. 1988). Recent model calculations made by Ferland et al. (1992) suggest that $\mathrm{O} v \mathrm{v}] \lambda 1218.39$ can become very strong in gas with a high ionization parameter, reaching $30 \%$ of the intensity of Ly $\alpha$. We were able to get a very good fit to the redward side of the Ly $\alpha$ spike in Q1451+1017 by including a 30\% contribution from O v] $\lambda 1218$. However, there then must be strong absorption in Ly $\alpha$ blueward of the line center; too many adjustable parameters are required for this to be a proof of the presence of $\mathrm{O} v] \lambda 1218$.

\section{A2. Q0000-398 AND Q2212-299}

These two objects both have moderately narrow emission lines. Within each object, all the emission lines can be fitted by the same profile except that $\mathrm{C}$ III] has a small amount of excess emission on its red shoulder. Overlying absorption at the position of $\mathrm{Ly} \beta$ in Q2212-299 causes the upper limit on its strength to be highly uncertain. In both QSOs there is a moderately strong line near $1300 \AA$ which gives a much better fit to $\mathrm{Si}$ II $\lambda 1307$ than to $\mathrm{O}_{\text {I }} \lambda 1303$; this is surprising since the $\mathrm{O}_{\mathrm{I}}$ line is expected to be strong as a result of fluorescence with Ly $\beta$. Both QSOs have significant amounts of $\mathrm{O}$ IV] $\lambda 1402$ contributing to the $\lambda 1400$ blend, although the exact split between O IV] and $\mathrm{Si}$ IV $\lambda 1397$ is quite uncertain. Al III and Fe II UV 191 are weak (but detectable) in both these objects, and the $\lambda 1909$ blend is dominated in both cases by C III].

\section{A3. $\mathrm{Q} 1623+268$}

This QSO has broader lines than any of the others studied here and profiles which are clearly different for different lines. Many of the line identifications are therefore uncertain. We again produced a template C IV $\lambda 1548.20$ profile by the same iterative technique employed with Q0207-398. The resulting profile gives a good fit to $\mathrm{N} v \lambda \lambda 1238.81,1242.80$, reproducing accurately the sharp drop to the redward side of the line center.

However, this template did not extend nearly far enough to the red to provide an adequate fit to $\mathrm{C}$ III] 21909 . The wavelength separation of the two highest peaks in the $\lambda 1909$ blend suggests that the main components are Si III] $\lambda 1892$ and $\mathrm{C}$ III] 21909 . Therefore, to obtain a template profile representing $\mathrm{C}$ III], we started again with a rough guess at the correct profile, fitted it to the $\mathrm{Al}$ III and $\mathrm{Si}$ III] lines in the blend, and then used the residual as an improved estimate of the $\mathrm{C}$ III] profile. The final C III] profile has FWHM $=3100 \mathrm{~km} \mathrm{~s}^{-1}$ (vs. $4900 \mathrm{~km} \mathrm{~s}^{-1}$ for C IV) and has its peak displaced $1900 \mathrm{~km} \mathrm{~s}^{-1}$ to the red of the C IV peak.

We tried fitting both these templates to various other lines in the spectrum. The fit to $\mathrm{O}$ vi is poorer than expected, but fitting C III] $\lambda 1909$ to O VI or either line to Ly $\beta$ gave significantly worse fits. Using the high-resolution data of Sargent, Boksenberg, \& Steidel (1988) as a guide to where the emission line is free of overlying absorption, we found that we could not get a reasonable fit to Ly $\alpha$ unless we constructed a profile made up of the C IV and C III] templates in roughly equal proportions. We could not tell whether the strong $\lambda 1400$ feature is O IV] or Si IV (or a combination of the two) because the velocity off set between the two available templates is close to the velocity difference between the two possible features. The results for the feature near $1305 \AA$ are similarly ambiguous; we cannot tell whether it is O I $\lambda 1303$ or Si II $\lambda 1307$.

Table 4 lists the results of our attempts to fit each of two different template profiles at the location of each line listed in Table 3. The columns headed " $\mathrm{A}$ " and " $\mathrm{B}$ " are for fits using the $\mathrm{C}$ IV and $\mathrm{C}$ III] templates respectively. 


\section{A4. $\mathrm{H} 0335-336$}

This QSO has extremely narrow emission lines (FWHM $\left.\sim 1000 \mathrm{~km} \mathrm{~s}^{-1}\right)$. Unfortunately, BAL absorption greatly confuses the line measurements shortward of $C_{\text {IV }} \lambda 1549$. To the red of $C$ IV, all lines can be fitted by a single profile; we used Fe II UV 191 as the template. Most of the line strength measurements in this region are taken from Hartig \& Baldwin (1986).

We then proceeded on the assumption that this would also be the correct template for the line profiles to the blue of C IV 21549. The line strengths listed in Table 4 for Si IV $\lambda 1397$ and $\mathrm{C}$ IV come from fitting just the red wing of the predicted blend, in which over half the flux from the blend must be absorbed away by the BAL system. The $\lambda 1400$ feature is identified with Si IV $\lambda 1397$ rather than O IV] $\lambda 1402$ because the observed emission does not extend nearly far enough to the red to be the latter. The strength of $\mathrm{C}$ II $\lambda 1335$ is estimated from fitting to the blue side of a narrow spike; the red side is eaten away by the blueward edge of the S IV BAL trough.

The region around Ly $\alpha$ is particularly messy. There are obvious peaks at the positions of $N v \lambda 1240$ and Si II $\lambda 1263$, but the highest point in the vicinity of Ly $\alpha$ is $600 \mathrm{~km} \mathrm{~s}^{-1}$ to the red of the expected position. There appears to be absorption at the expected line center, which could be causing the displacement of the peak. However, for any reasonable fit to the continuum (see Fig. 1), Ly $\alpha$ also appears to have an immense red tail which initially falls off rapidly until it reaches half-peak intensity at about $3500 \mathrm{~km} \mathrm{~s}^{-1}$, and then it drops more slowly to reach zero intensity at about $16,000 \mathrm{~km} \mathrm{~s}^{-1}$. This could be emission from outward-flowing gas on the far side of the nucleus, corresponding to the gas on the near side which produces the BAL systems. The $\mathrm{C}$ IV BAL system in this QSO has an unusually narrow trough extending to $-3300 \mathrm{~km} \mathrm{~s}^{-1}$, and then further absorption which, if $\mathrm{C}$ IV, extends clear to $-24,000 \mathrm{~km} \mathrm{~s}^{-1}$.

\section{A5. Q03408-4505}

In spite of the great similarity to $\mathrm{H} 0335$ - 336, the spectrum of this object was much harder to measure because of small but definite differences in the line profiles. We again used Fe II UV 191 as our template because it appears to have fewer problems with blending or BAL absorption than any of the other lines. We obtained reasonably good fits to $\mathrm{Al}$ III $\lambda 1857, \mathrm{C}$ II $\lambda 1335$, and $\mathrm{Si}$ IV $\lambda 1402.77$ (assuming that the companion Si IV $\lambda 1393.76$ line falls in the $\lambda 1402.77$ BAL trough). However, N v $\lambda 1240, \mathrm{Si}$ II $\lambda 1263, \mathrm{O}_{\text {I }} \lambda 1303, \mathrm{Si}$ II $\lambda 1307, \mathrm{C}$ IV $\lambda 1549$, the lines in the $\lambda 1909$ blend, and Mg II $\lambda 2798$ all appear to have somewhat broader profiles. The values in Table 4 for most of these broader lines are just rough guesses at how to divide up fluxes in blends and to allow for the effects of BAL absorption, made using both the $\lambda 1787.2$ of the $\mathrm{Mg}$ II $\lambda 2798$ profiles as guides.

The separation of the individual peaks in the $\lambda 1909$ blend are more consistent with $\mathrm{Fe}$ III than with Si III], C III]. In view of the great similarity to the spectrum of $\mathrm{H} 0335-337$, we have asigned all the flux from this blend to Fe III and listed upper limits for Si III] and C III] which are half the Fe III strength. Intermediate combinations of the relative line strengths are also acceptable, but we doubt that $\mathrm{Fe}$ III could be more than $50 \%$ weaker than the value given in Table 4.

\section{APPENDIX B}

\section{NUMERICAL DETAILS}

The following sections describe some details and assumptions used in the CLOUDY photoionization code (Ferland 1995).

\section{B1. ATOMIC DATA}

\section{B1.1. Data for $\mathrm{C}, \mathrm{N}, \mathrm{Al}$, and $\mathrm{Si}$ Lines}

Intensities of intercombination lines of nitrogen, aluminum, and silicon are crucial to this paper. Collision strengths for $\mathrm{N}$ III $\lambda 990$ and N III] $\lambda 1750$ are taken from Blum \& Pradhan (1992), and transition probabilities are from Stafford, Hibbert, \& Bell (1993). For N IV] $\lambda 1486$, the transition probabilities are taken from Allard et al. (1990), while the collision strengths are from Keenan et al. (1986). The Al III $\lambda 1860$ data are from Dufton et al. (1986) and Dufton \& Kingston (1987b). Collision strengths and transition probabilities for Si III] $\lambda 1892$ are taken from Dufton \& Kingston (1989) and Nussbaumer (1986), respectively. For Si IV 21397 , data are from Dufton \& Kingston (1987a) and Mendoza (1983). A series of permitted transitions of Si II are observed. We adopt the oscillator strengths given by Morton, York, \& Jenkins (1988) and the collision strengths of Dufton \& Kingston (1991). Recently, Kwong et al. (1993) measured experimentally the transition probability of C III $\lambda 1909$ to be $25 \%$ larger than the value derived by Nussbaumer \& Storey (1978), the new value being $120.9 \mathrm{~s}^{-1}$. We adopted this newer value.

\section{B1.2. Dielectronic Recombination}

The state specific rate coefficient for decays from an autoionizing level $a$ above the ionization threshold of atom $X^{+m}$ to the bound level $b$ of the atom is related to the abundance of the ion $X^{+m+1}$ by (Nussbaumer \& Storey 1983)

$$
\alpha_{\text {diel }}(a, T)=A_{a, b} b\left(X_{a}^{+m}\right) \frac{n^{*}\left(X_{a}^{+m}\right)}{n_{e}^{*} n^{*}\left(X_{a}^{+m+1}\right)}
$$

where $A_{a, b}$ is the rate coefficient for radiative decays to the bound level, $b\left(X_{a}^{+m}\right)$ is the departure coefficient for the autoionizing level, and the densities are their values in thermodynamic equilibrium. We assume that the autoionizing level is held in LTE by detailed balance between dielectronic recombination and autoionization. Converting the Einstein $A_{k i}$ to an oscillator 
strength $f_{i k}$, this expression becomes

$$
\begin{aligned}
\alpha_{\mathrm{diel}}(a, T) & =A_{a, b} b\left(X_{a}^{+m}\right) \frac{n^{*}\left(X_{a}^{+m}\right)}{n_{e}^{*} n^{*}\left(X_{a}^{+m+1}\right)}=\frac{g_{a}}{g_{e} g_{g}}\left(\frac{h^{2}}{2 \pi m k T}\right)^{3 / 2} \frac{8 \pi^{2}}{m c \lambda_{\mathrm{cm}}^{2}} \frac{g_{l}}{g_{a}} f_{\mathrm{abs}} \exp \left(\frac{-E_{a}}{k T}\right) \\
& \approx 1.38 \times 10^{-8} T^{-3 / 2} f_{\mathrm{abs}} \frac{g_{l}}{g_{\text {ion }}} \lambda_{\mu \mathrm{m}}^{-2} \exp \left(\frac{-E_{a}}{k T}\right),
\end{aligned}
$$

where the symbols have their usual meanings, the wavelength is expressed in microns, and we assume that the partition function of the ion is equal to the statistical weight of the ground state.

Dielectronic recombination rate coefficients for nitrogen are taken from Nussbaumer \& Storey (1983). These have not been computed for most third row elements, so means of rate coefficients derived for second row elements are used for third row elements (Al and Si included), as discussed by Ali et al. (1991). In addition, we note that the dielectronic recombination rates are accurate at low densities only; possible effects of high densities on these rates are uncertain. We use the fits from Davidson (1975). This uncertainty has a significant impact on the prediction of those emission lines for which dielectronic recombination is important (e.g., $\mathrm{N}$ III and Si II).

\section{B1.3. Atomic Line Acceleration}

The Opacity Project (OP) calculations (Seaton et al. 1992) produced a set of accurate atomic data for all stages of ionization of astrophysically important elements: $Z \leq 14$ and $Z=16,18,20,26$, where $Z$ is atomic number. In particular, the OP data include oscillator strengths for all optically allowed transitions between states with $n \leq 10$ and $1 \leq 4$. We retrieved from the OP database TOPbase (Cunto et al. 1993) the $g$-values for all multiplets which involve the ground term. These 6018 resonance multiplets include 23,505 lines. We calculated the $f$-values of all individual lines in multiplets based on $L S$ coupling rules (Russell 1936). The TOPbase does not include the data for Fe I and Fe II. We obtained the $f$-values for 109 lines of 17 resonance multiplets of $\mathrm{Fe}$ I from Fuhr, Martin, \& Wiese (1988) and 225 lines of 19 resonance multiplets of Fe II from Nahar (1995). In the whole, our line database includes the $f$-values of 23,839 lines of 6054 resonance multiplets.

These lines are treated as averaged multiplets rather than individual lines in the calculations described here. Each line has an associated optical depth, and the depth-dependent continuum pump rate is modeled as in Ferland (1992). The form of this function does not strongly affect results, since most line driving occurs over the first few optical depths, as the line absorbs the Doppler core.

\section{B1.4. Other Details}

All lines, including the intercombination lines, can become optically thick at the flux levels considered in this paper. All lines predicted here are transferred using escape probabilities, including destruction by the continuous background opacity. For strong resonance lines, interpolation on the results of Hummer \& Kunasz (1980) are used, and Hummer's K2 function (Hummer 1968, 1981) is used for lines characterized by complete redistribution in a Doppler core.

Secondary ionization efficiencies are taken from the recent calculations of Xu \& McCray (1991). Continuum fluorescence is an important excitation mechanism for many of the strong resonance lines in the component with large line width. This process is treated adopting the formalism presented by Ferland (1992).

\section{B2. MODEL PARAMETERS}

\section{B2.1. The Cloud Composition}

The $Z=5 Z_{\odot}$ mixture is that described in Paper II and is the lowest metallicity mixture capable of reproducing the limit to $\mathrm{Nv} / \mathrm{He}$ II in component $\mathrm{B}$ in Q0207-398. The specific $5 Z_{\odot}$ abundances used are, by number relative to hydrogen, as follows: $\mathrm{He}, 1.24 \times 10^{-1} ; \mathrm{Li}, 1.19 \times 10^{-8} ; \mathrm{Be}, 1.52 \times 10^{-10} ; \mathrm{B}, 4.45 \times 10^{-9} ; \mathrm{C}, 8.24 \times 10^{-4} ; \mathrm{N}, 1.15 \times 10^{-3} ; \mathrm{O}, 5.95 \times 10^{-3} ; \mathrm{F}$, $1.95 \times 10^{-7} ; \mathrm{Ne}, 7.94 \times 10^{-4} ; \mathrm{Na}, 1.36 \times 10^{-5} ; \mathrm{Mg}, 2.51 \times 10^{-4} ; \mathrm{Al}, 1.93 \times 10^{-5} ; \mathrm{Si}, 2.29 \times 10^{-4} ; \mathrm{P}, 2.41 \times 10^{-6} ; \mathrm{S}$, $1.06 \times 10^{-4} ; \mathrm{Cl}, 1.21 \times 10^{-6} ; \mathrm{Ar}, 2.34 \times 10^{-5} ; \mathrm{K}, 8.72 \times 10^{-7} ; \mathrm{Ca}, 1.42 \times 10^{-5} ; \mathrm{Sc}, 1.73 \times 10^{-9} ; \mathrm{Ti}, 1.22 \times 10^{-7} ; \mathrm{V}$, $1.49 \times 10^{-8} ; \mathrm{Cr}, 6.87 \times 10^{-7} ; \mathrm{Mn}, 4.85 \times 10^{-7} ; \mathrm{Fe}, 6.64 \times 10^{-5} ; \mathrm{Co}, 3.18 \times 10^{-9} ; \mathrm{Ni}, 2.50 \times 10^{-6} ; \mathrm{Cu}, 2.65 \times 10^{-8} ; \mathrm{Zn}$, $6.41 \times 10^{-8}$.

\section{B2.2. The Incident Continuum}

We use the continuum adopted in Paper II. Briefly, this was a big bump represented by $f_{v} \propto e^{-h v / E_{\text {cut }}}$ with an energy cutoff of $21.6 \mathrm{eV}$, added to a $f_{v} \propto v^{-1}$ X-ray power law extending to $100 \mathrm{keV}$. The two continua were normalized by a choice of $\alpha_{o x}=-1.2$. The choice of $\alpha_{o x}$ was driven by the need to match the observed $W_{\lambda}(\mathrm{N} v)$ in component $\mathrm{B}$ with a reasonable covering factor (0.2). The observed values were much smaller ( -1.57 and -1.84 ; Wilkes et al. 1994, Bechtold et al. 1994), and we concluded that the gas in component B may be viewing a continuum different from the observed one. If this is the case, then it is possible that component A sees yet another continuum. However, for reasons of simplicity and because the predictions of the general conditions within component A based upon the lower ionization emission lines will not be very sensitive to the continuum shape, we have chosen to use the same continuum shape here. See Paper II for more details concerning the choice of continuum shape.

\section{B2.3. Stopping Criterion}

The models were stopped when the electron density fell to 0.5 times the total hydrogen density. For the final component A model, the total hydrogen column density was $\sim 10^{23} \mathrm{~cm}^{-2}$, so the maximum depth considered was $\sim 10^{23} / 10^{12.65} \approx$ $10^{10.4} \mathrm{~cm}$. 


\section{APPENDIX C}

\section{THE Si II DISASTER}

Figure 10 shows a Grotrian diagram for the lowest levels of $\mathrm{Si}^{+}$, and selected terms of $\mathrm{Fe}^{+}$. Table 5 shows that our calculations explain adequately the intensity of the lowest $\mathrm{Si}$ II line, $\lambda 1814$, relative to $\mathrm{Al}$ III $\lambda 1857$, but they fail to reproduce the more highly excited Si II lines $(\lambda 1263, \lambda 1307)$ by $1-2$ orders of magnitude. These high-excitation lines are relatively strong and well measured: $\mathrm{Si}$ II $\lambda 1263 / \mathrm{Si}$ II $\lambda 1814=6$ and $\mathrm{Si}$ II $\lambda 1307 / \mathrm{Si}$ II $\lambda 1814=7$. We calculated grids of the $\mathrm{Si}$ II $\lambda 1307 / \mathrm{Si}$ II $\lambda 1814$ intensity ratio like those shown in Figure 7 for other lines and find that nowhere on the density-ionizing flux plane does the predicted $\mathrm{Si}$ II $\lambda 1307 / \mathrm{Si}$ II $\lambda 1814$ ratio come any closer than an order of magnitude to the observed one. This demonstrates that slight changes in parameters cannot help match this line ratio. In the work described above, we did not attempt to match any $\mathrm{Si}$ II line because of this fundamental uncertainty in the origin of the spectrum. Nevertheless, the final model (Table 5) reproduces the intensity of the lower excitation $\mathrm{Si}$ II line $(\lambda 1814)$ quite well. The problem is that the high-excitation lines are much stronger relative to $\lambda 1814$ than expected for collisional excitation.

This suggests that a selective excitation process, not included in the present calculations, affects excited levels of $\mathrm{Si}^{+}$. A question that may be related to this is the origin of Fe II UV 191 (also shown in Fig. 10). This multiplet is known to be selectively excited in stars (see the review by Johansson \& Hansen 1988), either by the continuum through UV 9, or by the chance coincidence that Fe II UV 9 overlaps with Si II $\lambda 1263$. This second line is, in fact, strong in our source, although our simulations cannot reproduce it. The sources with strong UV 191 also have strong Si II $\lambda 1263$. In fact, the Si II $\lambda 1263$ is stronger than the feature that is close to UV 191, suggesting that it is energetically possible for a fluorescence processes involving Si II to excite UV 191 with the observed intensity.

We have investigated several selective excitation processes to try to reproduce the intensity of the highly excited Si II lines. $\mathrm{Si}^{+}$has an autoionizing level very close to the ionization edge $\left(3 d^{2} F^{o}\right.$, shown in Fig. 10; Bashkin \& Stoner 1975). The autoionizing level decays to a bound transition through dielectronic recombination lines at $\lambda 1909$ and $\lambda 1307$, and features at these wavelengths are in fact strong (although we do not identify them with the stabilizing transition). We know of no estimates of the oscillator strength of the dielectronic recombination transitions to bound levels, so we assume unity. The result for the model presented in Table 5 is that the two transitions from the autoionizing level are predicted to have intensities roughly equal to the $\mathrm{Al}$ III line. We conclude that dielectronic recombination could be a competitive process in selectively exciting the $\lambda 1263$ and $\lambda 1814$ transitions of $\mathrm{Si}$ II. Unfortunately, we are not in a position to make reliable estimates of the dielectronic recombination rate coefficient, beyond what is described here. This process should be investigated further.

An alternative is charge transfer. $\mathrm{H}^{o}+\mathrm{Si}^{++} \rightarrow \mathrm{H}^{+}+\mathrm{Si}^{+}$is known to be fast, but the daughter is left in the ground state of $\mathrm{Si}^{+}$(Gargaud, McCarroll, \& Valiron 1982) and produces no Si II lines. We have investigated charge transfer into excited states of $\mathrm{Si}^{+}$, an energetically possible channel for the temperatures in our simulations. We assume a rate coefficient equal to that quoted by Gargaud et al. for transfer into ground, and we confirm that this would result in significant emission. This too should be investigated further.

For pure collisional excitation, the $\mathrm{Si}$ II $\lambda 1531$ line, which we do not see (but the line is blended with $\mathrm{C}$ IV), will have an intensity comparable to the higher excitation $\mathrm{Si}$ II lines such as $\lambda 1307$ and $\lambda 1263$. If $\lambda 1531$ is much weaker than $\lambda 1263$ or $\lambda 1307$, then this would be evidence that the latter lines are not collisionally excited.

It is because of the extreme conditions in component A that such processes as dielectronic recombination or charge transfer can produce significant emission. All resonance lines from this region are saturated near the blackbody limit because of thermalization at the high density. As a result, lines such as Al III are not efficient emitters, and processes which produce excited transitions such as the dielectronic recombination described above, become competitive.

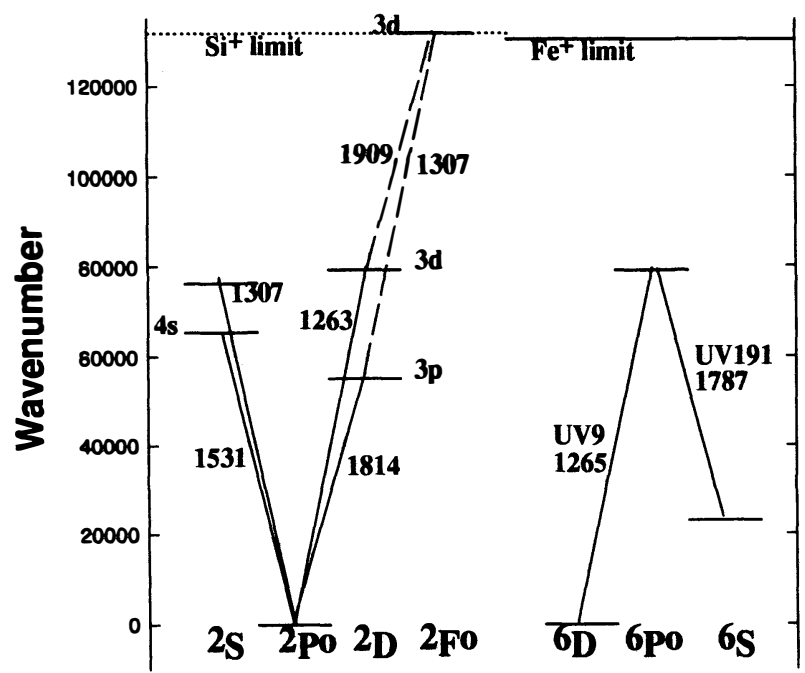

Fig. 10.-A partial Grotrian diagram for $\mathrm{Si}^{+}$and $\mathrm{Fe}^{+}$, with the lines discussed in this paper indicated 


\section{REFERENCES}

Alexander, T., \& Netzer, H. 1994, MNRAS, 270, 781

Ali, B., Blum, R. D., Bumgardner, T. E., Cranmer, S. R., Ferland, G. J., Haefner, R. I., \& Tiede, G. P. 1991, PASP, 103, 1182

Allard, N., Artu, M.-C., Lanz, T., \& Le Dourneuf, M. 1990, A\&AS, 84, 563

Baldwin, J., Ferland, G., Korista, K., \& Verner, D. 1995, ApJ, 455, L119

Baldwin, J. A., McMahon, R., Hazard, C., \& Williams, R. E. 1988, ApJ, 327,103

Bashkin, S., \& Stoner, J. O. 1975, Atomic Energy Levels and Grotrian Diagrams, Vol 1 (Amsterdam: North-Holland)

Bechtold, J., et al. 1994, AJ, 108, 374

Blum, R. D., \& Pradhan, A. K. 1992, ApJS, 80, 425

Boyle, B. J. 1990, MNRAS, 243, 231

Boyle, B. J., Fong, R., Shanks, T., \& Peterson, B. A. 1990, MNRAS, 243, 1

Brotherton, M. S., Wills, B. J., Francis, P. J., \& Steidel, C. C. 1994, ApJ, 430, 495

Corbin, M. R. 1990, ApJ, 357,346

Cunto, W., Mendoza, C., Ochsenbein, F., \& Zeippen, C. J. 1993, A\&A, 275, L5

Davidson, K. 1975, ApJ, 195, 285

Dufton, P. L., \& Kingston, A. E. 1989, MNRAS, 241, 209

1987a, J. Phys. B, 20, 2899

1987b, J. Phys. B, 20, 3899

1991, MNRAS, 248, 827

Dufton, P. L., et al. 1986, MNRAS, 222, 713

Edwards, A.C. 1980, MNRAS, 190,757

Espey, B. R., Carswell, R. F., Bailey, J. A., Smith, M. G., \& Ward, M. J. 1989, ApJ, 342, 666

Ferguson, J. W., Ferland, G. J., \& Pradhan, A.K. 1994, ApJ, 438, L55

Ferland, G. J. 1992, ApJ, 389, L63

1995, Hazy, a Brief Introduction to Cloudy (Univ. Kentucky Phys. Dept. Internal Rep.)

Ferland, G. J., Baldwin, J. A., Korista, K. T., Hamann, F., Carswell, R. F. Phillips, M. M., Wilkes, B. J., \& Williams, R. E. 1996, ApJ, 461, 683 (Paper II)

Francis, P. J., Hewet, P. C., Foltz, C. B., \& Chaffee, F. H. 1992, ApJ, 398, 476

Fuhr, J. R., Martin, G. A., \& Wiese, W. L. 1988, J. Phys. Chem. Ref. Data, 17, Suppl. 4

Gaskell, C. M. 1982, ApJ, 263, 79

Gaugaud, M., McCarroll, R., \& Valiron, P. 1982, A\&A, 106, 197

Graham, M. J.Clowes, R. G. \& Campusano, L. E. 1995, MNRAS, in press

Hamann, F., Korista, K. T., \& Morris, S. L. 1993, ApJ, 415, 541

Harms, R. J., et al. 1994, ApJ, 435, L35

Hartig, G. F., \& Baldwin, J. A. 1986, ApJ, 302,64

Hazard, C., Morton, D. C., Terlevich, R., \& McMahon, R. G. 1984, ApJ, 282,33
Hummer, D. G. 1968, MNRAS, 138, 73

1981, J. Quant. Spectrosc. Radiat. Transfer, 26, 187

Hummer, D. G., \& Kunasz, P. B. 1980, ApJ, 236, 609

Johansson, S., \& Hansen, J. E. 1988, in Physics of Formation of $\mathrm{Fe}$ II Lines Outside LTE, ed. R. Viotti et al.(Dordrecht: Reidel), 235

Junkkarinen, V.T., Burbidge, E. M., \& Smith, H. E. 1983, ApJ, 265, 51

Kazanas, D. 1989, ApJ, 347, 74

Keenan, F. P., Berrington, K. A., Burke, P. G., Dufton, P. L., \& Kingston, A. E. 1986, Phys. Scripta, 34, 216

Kwong, V. H. S., Fang, Z., Gibbons, T. T., Parkinson, W. H., \& Smith, P. L. 1993, ApJ, 411, 431

Mathews, W.G.1983, ApJ, 272, 390

Mendoza, C. 1983, in IAU Symp. 103, Planetary Nebulae, ed. D. R. Flower (Dordrecht: Reidel), 143

Morton, D. C., York, D. G., \& Jenkins, E. B. 1988, ApJS, 68, 449

Nahar, S. N. 1995, A\&A, 293, 967

Nussbaumer, H. 1986, A\&A, 155, 205

Nussbaumer, H., \& Storey, P. J. 1978, A\&A, 64, 139

. 1983, A\&A, 126,75

Osterbrock, D. E. 1989, Astrophysics of Gaseous Nebulae and Active Galactic Nuclei (Mill Valley: University Science Press)

. 1991, Rep. Prog. Phys., 54, 579

Penston, M. V. 1988, MNRAS, 233, 601

Perry, J. J., \& Dyson, J. E. 1985, MNRAS, 213, 665

Roos, N. 1992, ApJ, 385, 108

Russell, H. N. 1936, ApJ, 83, 129

Sargent, W. L. W., Boksenberg, A. \& Steidel, C. C. 1988, ApJS, 68, 539

Sargent, W. L. W., Young, P. J., Boksenberg, A., Shortridge, K., Lynds, C. R., \& Hartwick, F. D. A. 1978, ApJ, 221, 731

Scoville, N. Z., \& Norman, C. 1988, ApJ, 332, 163 1995, ApJ, 451, 510

Seaton, M. J. 1960, Rep. Prog. Phys., 23, 313

Seaton, M. J., et al. 1992, Rev. Mexicana Astron. Astrofis., 23, 19

Stafford, R. P., Hibbert, A. \& Bell, K. L. 1993, MNRAS, 260, L11

Steidel, C. C., \& Sargent, W. L. W. 1991, ApJ, 382, 433

Ulrich, M.-H. 1989, A\&A, 220, 71

Voit, G. M., \& Shull, J. M. 1988, ApJ, 331, 197

Weymann, R. J., Morris, S. L., Foltz, C. B., \& Hewett, P. C. 1991, ApJ, 373,

Wilkes, B. J. 1984, MNRAS, 207, 73

Wilkes, B. J., Tananbaum, H., Worrall, D. M., \& Avni, Y. 1994, ApJS, 92,

Wills, B. J., Brotherton, M. S., Fang, D., Steidel, C. C., \& Sargent, W. L. W. 1993, ApJ, 415, 563

Xu, Y., \& McCray, R. 1991, ApJ, 375, 190 